\title{
Slow dynamics of supercooled water confined in nanoporous silica materials
}

\author{
L Liu ${ }^{1}$, A Faraone ${ }^{1,2}$, C-Y Mou ${ }^{3}$, C-W Yen ${ }^{3}$ and S-H Chen ${ }^{1,4}$ \\ ${ }^{1}$ Department of Nuclear Engineering, 24-209 MIT, Cambridge, MA 02139, USA \\ 2 Department of Physics and INFM, University of Messina, Messina 98166, Italy \\ ${ }^{3}$ Department of Chemistry, National Taiwan University, Taipei, 106, Taiwan \\ E-mail: sowhsin@mit.edu
}

Received 27 February 2004

Published 29 October 2004

Online at stacks.iop.org/JPhysCM/16/S5403

doi:10.1088/0953-8984/16/45/007

\begin{abstract}
We review our incoherent quasielastic neutron scattering (QENS) studies of the dynamics of supercooled water confined in nanoporous silica materials. QENS data were analysed by using the relaxing cage model (RCM) previously developed by us. We first use molecular dynamics (MD) simulation of the extended simple point charge model (SPC/E) for bulk supercooled water to establish the validity of the RCM, which applies to both the translational and rotational motion of water molecules. We then assume that the dynamics of water molecules in the vicinity of a hydrophilic surface is similar to a bulk water at an equivalent lower supercooled temperature. This analogy was experimentally demonstrated in previous investigations of water in Vycor glasses and near hydrophilic protein surfaces. Studies were made of supercooled water in MCM-41-S (pore sizes 25, 18, and $14 \AA$ ) and MCM48-S (pore size $22 \AA$ ) using three QENS spectrometers of respective energy resolutions 1,30 , and $60 \mu \mathrm{eV}$, covering the temperature range from 325 to $200 \mathrm{~K}$. Five quantities are extracted from the analysis: they are $\beta$, the stretch exponent characterizing the $\alpha$-relaxation; $\beta \gamma$, the exponent determining the power-law dependence of the relaxation time on $Q ;\left\langle\tau_{0}\right\rangle$, the $Q$-independent pre-factor for the average translational relaxation time; $\left\langle\tau_{\mathrm{R}_{1}}\right\rangle$, the relaxation time for the first-order rotational correlation function; and $\left\langle\tau_{\mathrm{R}_{2}}\right\rangle$, the relaxation time for the second-order rotational correlation function. We discuss the temperature dependence of these parameters and note that, in particular, the dynamics is rapidly slowing down at temperature around $220 \mathrm{~K}$, signalling the onset of a structural arrest transition of liquid water into an amorphous solid water.
\end{abstract}

4 Author to whom any correspondence should be addressed. 


\section{Introduction}

The dynamical properties of bulk supercooled water have attracted a great deal of interest during the last decades; however, the details of the microscopic motions of water molecules in the deep supercooled region are still far from being understood. A large number of recent works focus on this topic [1-3]. It has been suggested that properties of supercooled water show power-law-like anomalies going toward a limiting temperature, $T_{\mathrm{s}} \sim 227 \mathrm{~K}[4]$. Nevertheless, an experimental detection of such a behaviour is not easy in practice since bulk water at ambient pressure can be supercooled only down to the homogenous nucleation temperature, $T_{\mathrm{H}} \sim 235 \mathrm{~K}$. However, one may bypass this homogeneous nucleation temperature barrier if water is confined in nanoporous matrices. On the other hand, water in many real systems is not in its bulk form but located in the vicinity of surfaces or filling small cavities, such as water in rocks, in polymer gels, in biological membranes, and in dense colloidal solutions [5]. Moreover, the dynamics of water is affected by the microstructure of the nanoporous materials, which is relevant in processes involving catalysis and separation. Therefore, the study of water in confined geometry is of fundamental importance for clarifying the basic properties of water and it is likely to be the key to understand many real systems. Experimentally, the structural properties of water in confinement [6] and at supercooled temperatures [7] were studied extensively using x-ray and neutron diffraction. On the other hand, the relaxational dynamics of water confined in mesoporous matrices were studied using dielectric spectroscopy [8-14] and different nuclear magnetic resonance (NMR) techniques [15-20].

Due to the fact that the incoherent scattering cross section of hydrogen is much larger than the sum of that of oxygen, silicon, and carbon, which mainly constitute the confining materials, incoherent quasielastic neutron scattering (QENS) is a well established and powerful method for studying the self-dynamics of the hydrogen atoms in a water molecule in bulk or in confinement. Moreover, by combining the results from time-of-flight (TOF) and backscattering spectrometers, it is possible to study the dynamics of water in a wide range of timescale, encompassing the picosecond and nanosecond range. At the same time, by investigating $Q$ values ( $Q$ being the magnitude of the wavevector transfer) in the range $0.2 \AA^{-1} \leqslant Q \leqslant 2.00 \AA^{-1}$, the spatial characteristics of water dynamics can be investigated at a sub-nanometre scale. Moreover, the experimentally determined self-dynamic structure factor is related to the intermediate scattering function (ISF) by a Fourier transform. This special feature allows the interpretation of the results obtained by the experiments in terms of a direct comparison with theory and MD data on the intermediate scattering functions.

Therefore, the QENS technique has been employed for the study of water dynamics in nanoporous matrices. We carried out molecular dynamics (MD) simulations and used different energy resolutions of the QENS spectrometers to study the dynamics of water in confined geometries covering a wide range of timescale. MD simulations of the extended simple point charge (SPC/E) model of water have furnished detailed and accurate results for understanding the dynamics of supercooled water [21-25]. From the analysis of these results, the relaxing cage models (RCMs) for the translational and rotational dynamics of water at supercooled temperatures have been developed. Moreover, MD simulations of SPC/E water confined in silica nanopores have been carried out as well in the literature [26,27]. The interaction between the hydrophilic surface and the water molecules has noticeable effects on the structure of the first and second layers of water near the pore surface [28]. As far as the dynamics is concerned, in confinement water molecules are in the glassy state with very low mobility and already have a non-exponential relaxation behaviour at ambient conditions. In general, the water molecules show a dynamics similar to that of supercooled water [26, 27]. 
Different nanoporous matrices, e.g. Vycor [29-32], GelSil [33, 34], clay [35, 36], and MCM-41 [37-40], have been used as confining media. Among the different nanoporous silica matrices, the relatively recently discovered MCM represents an interesting opportunity for studying water in confinement. For example, in comparison with Vycor glass, these molecular sieves have smaller pores with a narrower size distribution.

In this paper we will explain in detail the RCM theory and the MD results of dynamics of supercooled bulk water, and report the results of our QENS study on water confined in laboratory synthesized MCM-41-S and MCM-48-S. These MCM samples allow us to study dynamical effects of different pore morphologies; in fact, the MCM-41-S matrix has 1D cylindrical tubes arranged in a hexagonal structure whereas MCM-48-S has 3D bicontinuous morphology. It is very important to note that the small dimension of these pores allows us to investigate temperatures below the homogeneous nucleation temperature.

In general, the present study is a systematic investigation of the temperature behaviour of both the translational and rotational dynamics of water inside nanoporous materials, from room temperature down to the limiting temperature $T_{\mathrm{s}}$ of supercooled water. The data, collected on three different spectrometers, have been analysed according to a single consistent model (RCM), which we have developed in the past years. The obtained results show the exponential slowing down of both the translational and rotational dynamics.

\section{SPC/E simulation}

We performed MD simulations of 216 water molecules at temperatures $T=284.5,263.0$, 250.0, 225.0, 220.0 and 215.0 K, interacting via the SPC/E pair potential [41]. This is a more suitable method to test out our model of the decoupling approximation and rotational correlation functions than using real neutron scattering data, since MD data do not have the complication of the resolution effect as do real experimental data. We carried out an extensive simulation, in an $N V E$ ensemble with 216 water molecules contained in a cubic box of edge $18.65 \AA$. The effective potential used is the extended simple point charge model, SPC/E. This potential treats a single water molecule as a rigid set of point masses with an $\mathrm{OH}$ distance of $0.1 \mathrm{~nm}$ and an $\mathrm{HOH}$ angle equal to the tetrahedral angle $109.47^{\circ}$. The point charges are placed on the atoms and their magnitudes are $q_{\mathrm{H}}=0.4238 e$ and $q_{\mathrm{O}}=-2 q_{\mathrm{H}}=-0.8476 e$. Only the oxygen atoms in different molecules interact among themselves via a Lennard-Jones potential, with the parameters $\sigma=0.31656 \mathrm{~nm}$ and $\epsilon=0.64857 \mathrm{~kJ} \mathrm{~mol}^{-1}$. The interaction between pairs of molecules is calculated explicitly when their separation is less than a cut-off distance $r_{\mathrm{c}}$ of $2.5 \sigma$. The contribution due to Coulomb interactions beyond $r_{\mathrm{c}}$ is calculated using the reaction-field method, as described by Steinhauser [42]. Also, the contribution of LennardJones interactions between pairs separated by more than $r_{\mathrm{c}}$ is included in the evaluation of thermodynamic properties by assuming a uniform density beyond $r_{\mathrm{c}}$. A heat bath [43] has been used to allow for heat exchange when changing the temperature of the system. After the system has been equilibrated, the heat bath is then removed. In our simulations, periodic boundary conditions are used. The time step for the integration of the molecular trajectories is 1 fs. Simulations at low $T$ were started from equilibrated configurations at higher $T$. Equilibration was monitored via the time dependence of the potential energy. In all cases the equilibration time $t_{\mathrm{eq}}$ was longer than the time needed to enter the diffusive regime. We note that, for the SPC/E model of water, the density maximum occurs at about $250 \mathrm{~K}$, which corresponds to $277 \mathrm{~K}$ in real water. For the lower temperatures, 225-210 K, we recorded water trajectories for more than $1 \mathrm{~ns}$. For the other temperatures we recorded for $0.1 \mathrm{~ns}$. Further detailed thermodynamic parameters of the simulations are given in $[22,41]$. 
Table 1. Simulated state points.

\begin{tabular}{lllll}
\hline$T(\mathrm{~K})$ & $\rho_{\mathrm{s}}\left(\mathrm{g} \mathrm{cm}^{-3}\right)$ & $E\left(\mathrm{~kJ} \mathrm{~mol}^{-1}\right)$ & $P(\mathrm{MPa})$ & $D\left(10^{-5} \mathrm{~cm}^{2} \mathrm{~s}^{-1}\right)$ \\
\hline 284.5 & 0.984 & -48.1 & $-73 \pm 11$ & $(1.3 \pm 0.1) \times 10^{0}$ \\
263.0 & 0.985 & -49.4 & $-70 \pm 12$ & $(7.5 \pm 0.6) \times 10^{0}$ \\
250.0 & 0.986 & -50.0 & $-76 \pm 12$ & $(5.2 \pm 0.5) \times 10^{-1}$ \\
225.0 & 0.984 & -52.6 & $-75 \pm 15$ & $(4.4 \pm 0.4) \times 10^{-2}$ \\
220.0 & 0.984 & -53.1 & $-72 \pm 16$ & $(6.2 \pm 0.3) \times 10^{-3}$ \\
215.0 & 0.984 & -53.7 & $-75 \pm 18$ & $(1.2 \pm 0.1) \times 10^{-3}$ \\
\hline
\end{tabular}

In the investigated $Q$ range, the vibrational contribution drops out of consideration (explained later). Because of the above mentioned simplification, in a molecular dynamics simulation the QENS spectra can be obtained taking water to be effectively a rigid molecule using, for example, the SPC/E model potential.

The SPC/E potential has been explicitly parametrized to reproduce the experimental value of the self-diffusion constant at ambient temperature and at a density of $1 \mathrm{~g} \mathrm{~cm}^{-3}$ [43]. Densities in our simulation have been chosen on the basis of trial and error in preliminary runs. The corresponding pressures for the chosen final densities are reported in table 1, and this has been well described in [22].

\section{Relaxing cage model and mode-coupling theory}

We use ideas from mode-coupling theory (MCT) of supercooled liquids to formulate the relaxing cage model (RCM) [23]. The cage effect in the liquid state, which can be pictured as a transient trapping of molecules as a result of lowering temperature or increasing density, is a main idea in MCT [44, 45]. Microscopic density fluctuations of disordered high temperature and low density fluids usually relax rapidly in a timescale of a few picoseconds. Then, upon lowering the temperature below the freezing point or increasing the density of the liquid by applying a pressure, a rapid increase in the local order surrounding a particle, called a cage, leads to a substantial increase of the local structural relaxation time. A trapped particle in a cage, in the dense or supercooled liquid regime, can only migrate through rearrangement of a large number of particles surrounding it. Therefore, there is a strong coupling between the single-particle motion and the density fluctuations of the fluid. According to MCT, the long time cage structural relaxation behaviour is completely determined by the equilibrium structure factor $S(Q)$ of the liquid. It predicts that at the singular temperature, $T_{\mathrm{s}}$, the structural relaxation time becomes infinity and the supercooled liquid shows a phenomenon of structural arrest. On approaching $T_{\mathrm{s}}$ from above, there is a larger and larger separation between the timescales describing the rattling motion of a particle in the cage and the eventual structural relaxation time of the cage. Numerically, various model systems, such as the hard sphere system [46] or a mixed Lennard-Jones system [47], have shown this prediction and that the time evolution of the structural relaxation (called the $\alpha$ relaxation) is well approximated by a stretched exponential decay with a system dependent stretch exponent.

Upon supercooling, water undergoes an expansion or lowering of density. On lowering the temperature below the freezing point, water develops a tendency to form a hydrogenbonded, tetrahedrally coordinated first- and second-neighbour shells around a given molecule. Compared with five- or six-neighbour configurations which are known to be present with higher probability at higher temperatures, this configuration is a more open structure, so that the structural relaxation time of water increases rapidly upon supercooling since the 


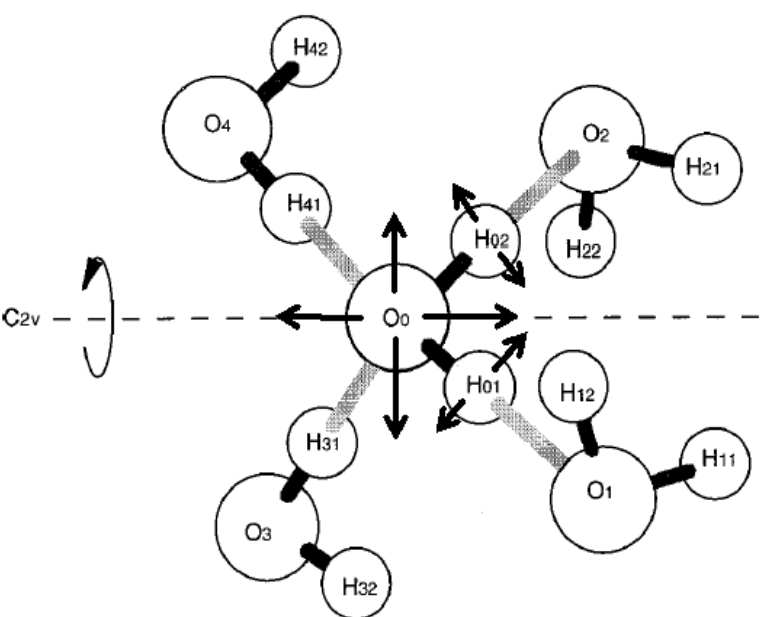

Figure 1. A schematic diagram for the hydrogen-bonded, tetrahedrally coordinated nearest neighbour cage in supercooled water.

tetrahedrally coordinated hydrogen-bonded structure, shown in figure 1 , is an inherently more stable structure locally and has a longer lifetime. At short times, less than $0.1 \mathrm{ps}$, the water molecule performs harmonic vibrations and librations inside the cage; at long times, longer than $1.0 \mathrm{ps}$, the cage eventually relaxes and the trapped particle can migrate through the rearrangement of a large number of particles surrounding it. Thus, the centre of mass motion of a supercooled water molecule can be considered as a compounded motion of a short time in-cage vibration and a long time cage relaxation, having two widely separated timescales. This is the so-called relaxing cage model. To analyse the translational and rotational dynamics of water at supercooled temperature, we have developed the RCM in the past few years. This model has been tested with MD simulations of SPC/E water [23, 24], and has been used to analyse QENS data [48-50].

\subsection{Dynamic structure factor}

Since the incoherent scattering cross section of hydrogen is roughly 20 times larger than the total scattering cross section of oxygen, silicon, and aluminium in the porous matrices, we may only take into account the contribution from the hydrogen atoms in the double differential scattering cross section of water and deal with the self-dynamic structure factor of the hydrogen atoms in the water molecules. In a quasielastic neutron scattering experiment, we can measure the double differential scattering cross section $\mathrm{d}^{2} \sigma_{\mathrm{H}} / \mathrm{d} \Omega \mathrm{d} \omega$, where $\sigma_{\mathrm{H}}$ is the incoherent scattering cross section of a hydrogen atom, $\mathrm{d} \Omega$ is the differential solid angle into which the neutron is scattered and $E=\hbar \omega$ is the energy transferred by the neutron to the sample. We have a well known relation

$$
\frac{\mathrm{d}^{2} \sigma_{\mathrm{H}}}{\mathrm{d} \Omega \mathrm{d} \omega}=2 N \frac{\sigma_{\mathrm{H}}}{4 \pi} \frac{k_{\mathrm{f}}}{k_{\mathrm{i}}} S_{\mathrm{H}}(Q, \omega)
$$

where $N$ is the number of water molecules in the sample, $k_{\mathrm{i}}$ and $k_{\mathrm{f}}$ are, respectively, the wavenumbers of the incident and scattered neutrons, and $S_{\mathrm{H}}(Q, \omega)$ is the self-dynamic structure factor. Since $N, \sigma_{\mathrm{H}}, k_{\mathrm{i}}$, and $k_{\mathrm{f}}$ are all known quantities in a quasielastic scattering experiment, $S_{\mathrm{H}}(Q, \omega)$ can be straightforwardly extracted from the double differential scattering cross section.

In van Hove theory of neutron scattering [51], $S_{\mathrm{H}}(Q, \omega)$ is given in terms of the Fourier transform of the intermediate scattering function (ISF) of the hydrogen atom, $F_{\mathrm{H}}(Q, t)$, 
according to the equation

$$
S_{\mathrm{H}}(Q, \omega)=\frac{1}{2 \pi} \int_{-\infty}^{\infty} \mathrm{d} t \mathrm{e}^{\mathrm{i} \omega t} F_{\mathrm{H}}(Q, t) .
$$

We can then see that $F_{\mathrm{H}}(Q, t)$ is the primary quantity of theoretical interest related to experiments. It can be calculated by a model, such as RCM, and molecular dynamics simulation of SPC/E model of water.

\subsection{Decoupling approximation}

The dynamics of a hydrogen atom is composed of three components: the vibrational motion of the atom around its equilibrium position, the rotational motion of the atom around the centre of mass, and the translational motion of the centre of mass. The decoupling approximation [52] has been generally assumed in the analysis of QENS data of water. In this approximation, the ISF of the hydrogen atoms is written as the product of the vibrational ISF, $F_{\mathrm{V}}(Q, t)$, rotational ISF, $F_{\mathrm{R}}(Q, t)$, and translational ISF, $F_{\mathrm{T}}(Q, t)$ :

$$
F_{\mathrm{H}}(Q, t)=F_{\mathrm{T}}(Q, t) \cdot F_{\mathrm{R}}(Q, t) \cdot F_{\mathrm{V}}(Q, t) .
$$

The vibrational contribution, or the inelastic contribution, can be well approximated by a Debye-Waller factor [53], $\exp \left[-\frac{1}{3}\left\langle u^{2}\right\rangle Q^{2}\right]$. This is because we are concerned only with analysis of neutron spectra in the quasi-elastic region $(0<E<3000 \mu \mathrm{eV})$, which is equivalent to a timescale of picoseconds or longer in the ISF. $\left\langle u^{2}\right\rangle$ is the mean square vibrational amplitude of the hydrogen atom around its equilibrium position. Since O-H distance in a water molecule is about $1 \AA, \sqrt{\left\langle u^{2}\right\rangle} \leqslant 0.1 \AA$. In the investigated $Q$ range $(Q<2 \AA)$, the vibrational Debye-Waller factor is essentially unity. This also implies the validity of the SPC/E model in simulating ISF by assuming a rigid water molecule. As the consequence, the ISF of the hydrogen atoms can be expressed as the product of the rotational and translational ISFs,

$$
F_{\mathrm{H}}(Q, t)=F_{\mathrm{T}}(Q, t) \cdot F_{\mathrm{R}}(Q, t) .
$$

\subsection{The validity of the decoupling approximation}

We start to discuss the validity of the decoupling approximation, equation (4), by defining a new function, $F_{\mathrm{CON}}(Q, t)$. From the definition of intermediate scattering function, $F_{\mathrm{H}}(Q, t)=\left\langle\mathrm{e}^{-\mathrm{i} \vec{Q} \cdot(\vec{r}(t)-\vec{r}(0))}\right\rangle$, where $\vec{r}(t)$ is the position of the hydrogen atom at time $t$. Since $\vec{r}(t)=\vec{R}(t)+\vec{b}(t)$, where $\vec{b}(t)$ denotes a vector from the centre of mass to the hydrogen atom and $\vec{R}(t)$ denotes the position of the centre of mass, we can rewrite $F_{\mathrm{H}}(Q, t)$ as the product of four factors,

$$
F_{\mathrm{H}}(Q, t)=\left\langle\mathrm{e}^{-\mathrm{i} \vec{Q} \cdot \vec{R}(0)} \mathrm{e}^{-\mathrm{i} \vec{Q} \cdot \vec{b}(0)} \mathrm{e}^{\mathrm{i} \vec{Q} \cdot \vec{R}(t)} \mathrm{e}^{\mathrm{i} \vec{Q} \cdot \vec{b}(t)}\right\rangle .
$$

When dealing with a correlation function that is a product of four terms, each one with a $(Q, t)$ dependence, it is generally possible to rewrite it as the sum of all the possible binary factorizations of its terms plus another irreducible term, which we now call the connected intermediate scattering function $F_{\mathrm{CON}}(Q, t)$,

$$
\begin{aligned}
& F_{\mathrm{H}}(Q, t)=\left\langle\mathrm{e}^{-\mathrm{i} \vec{Q} \cdot \vec{R}(0)} \mathrm{e}^{\mathrm{i} \vec{Q} \cdot \vec{R}(t)}\right\rangle \times\left\langle\mathrm{e}^{-\mathrm{i} \vec{Q} \cdot \vec{b}(0)} \mathrm{e}^{\mathrm{i} \vec{Q} \cdot \vec{b}(t)}\right\rangle \\
& +\left\langle\mathrm{e}^{-\mathrm{i} \vec{Q} \cdot \vec{R}(0)} \mathrm{e}^{\mathrm{i} \vec{Q} \cdot \vec{b}(t)}\right\rangle \times\left\langle\mathrm{e}^{\mathrm{i} \vec{Q} \cdot \vec{R}(t)} \mathrm{e}^{-\mathrm{i} \vec{Q} \cdot \vec{b}(0)}\right\rangle+F_{\mathrm{CON}}(Q, t) .
\end{aligned}
$$

The time dependence of $\vec{b}(t)$ is independent of the choice of the reference system. In the reference system defined by the molecular centre of mass $\vec{R}(t)$, all mixed correlation functions vanish. Therefore, the contributions arising from all the terms composed of products 




Figure 2. The intermediate scattering functions (ISFs) at three $Q$ values $\left(0.75,1.51\right.$, and $\left.2.26 \AA^{-1}\right)$ and at $T=225 \mathrm{~K}$, as a function of time in a logarithmic scale. The open circles represent $F_{\mathrm{H}}(Q, t)$, the top solid curves, $F_{\mathrm{CM}}(Q, t) \times F_{\mathrm{R}}(Q, t)$, the dash-dot curve, the connected part of the correlation function, $F_{\mathrm{CON}}(Q, t)$, and the thick solid curve, the difference, $F_{\mathrm{CM}}(Q, t)-F_{\mathrm{H}}(Q, t)$. It is to be noted that at low $Q$ the decoupling approximation is good, but at high $Q$ the approximation progressively becomes poorer at long time but the deviation never exceeds 0.09 . However, it is also noticeable that at long time $(t>1 \mathrm{ps}) F_{\mathrm{H}}$ nearly coincides with $F_{\mathrm{CM}}$.

of $\overrightarrow{\boldsymbol{R}}$ and $\overrightarrow{\boldsymbol{b}}$ variables at an arbitrary time are zero on average. Generally speaking, $F_{\mathrm{CON}}(Q, t)$ is different from zero and contains the contribution coming from the four factors coupled together in the correlation function. Therefore, we can get the following relation:

$$
F_{\mathrm{H}}(Q, t)=F_{\mathrm{T}}(Q, t) F_{\mathrm{R}}(Q, t)+F_{\mathrm{CON}}(Q, t)
$$

where $F_{\mathrm{CON}}(Q, t)$ describes the strength of the coupling between translational and rotational motions as a function of $Q$ and $t$, as observed by QENS. Even though the rotational and translational motions of a water molecule are strongly coupled at all times [24, 25], MD simulations of SPC/E water at supercooled temperature have shown that the decoupling approximation is good to a few per cent.

In the graphs of figure 2 we show in a semi-logarithmic scale the following four quantities: $F_{\mathrm{H}}(Q, t), F_{\mathrm{CM}}(Q, t) \times F_{\mathrm{R}}(Q, t), F_{\mathrm{CON}}(Q, t)$, and $F_{\mathrm{CM}}(Q, t)-F_{\mathrm{H}}(Q, t)$. These functions are shown for a temperature $225 \mathrm{~K}$ at three $Q$ values. These $Q$ values are also quite close to the maximum and the minimum $Q$ value that can be probed by a typical QENS experiment. We see that $F_{\mathrm{H}}(Q, t)$ has the same short time features as $F_{\mathrm{CM}}(Q, t) \times F_{\mathrm{R}}(Q, t)$ but the same long time feature as $F_{\mathrm{CM}}(Q, t)$, so that $F_{\mathrm{CON}}(Q, t)$ is very small at time smaller than 1 ps but 


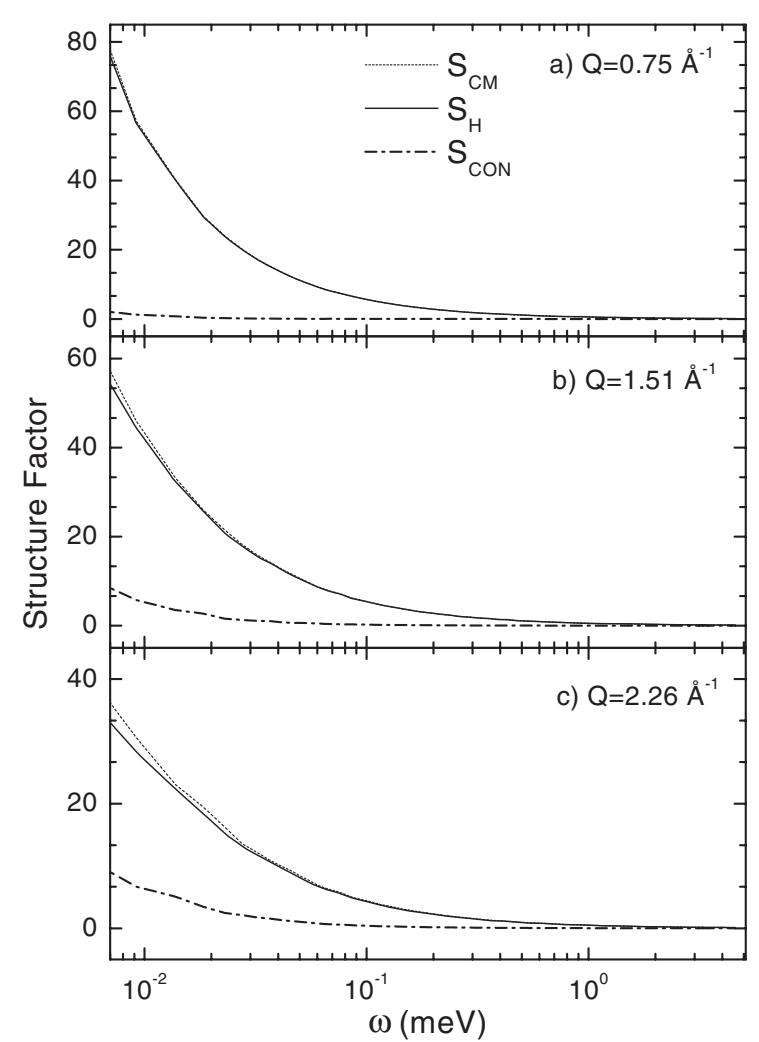

Figure 3. The dynamic structure factor at three $Q$ values $\left(0.75,1.51\right.$, and $\left.2.26 \AA^{-1}\right)$ and at $T=225 \mathrm{~K}$, as a function of frequency in a logarithmic scale. Also note the Fourier transform of the fit to the intermediate scattering functions of the centre of mass and the hydrogen atoms and the direct Fourier transform of the connected correlation contribution. The solid curves represent $S_{\mathrm{H}}(Q, \omega)$, the dashed curves, $S_{\mathrm{CM}}(Q, \omega)$, and the dash-dot curve, the connected part of the structure factor, $S_{\mathrm{CON}}(Q, \omega)$. It is to be noted that only at low frequency, $S_{\mathrm{CM}}(Q, \omega)$ is different from $S_{\mathrm{H}}(Q, \omega)$ by showing a higher peak. This difference increases as $Q$ gets bigger, but never exceeds 0.09 .

becomes non-negligible for long time. In contrast $F_{\mathrm{CM}}(Q, t)-F_{\mathrm{H}}(Q, t)$ is negligible at time longer than 1 ps but large at short time. Both $F_{\mathrm{CON}}(Q, t)$ and $F_{\mathrm{CM}}(Q, t)-F_{\mathrm{H}}(Q, t)$ increase substantially with increasing $Q$ value, but never reach 0.09 in magnitude.

In the graphs of figure 3 , we show that the coupling of rotational and translational motions is in general non-negligible for high $Q$ values. In the frequency space, the difference between the centre of mass and the hydrogen is not as big as the contribution from $F_{\mathrm{CON}}(Q, t)$.

\subsection{Theory for the translational intermediate scattering function}

Having established the validity of the decoupling approximation, equation (4), we now discuss how to get the translational and the rotational intermediate scattering functions separately. For the translational ISF, the relaxing cage picture gives us an idea to express $F_{\mathrm{T}}(Q, t)$ in terms of the product of the short time dynamics and a long time decay of the ISF. This is because the timescale for the in-cage vibrational motion and the long time relaxation of the cage itself are well separated in the supercooled water. We first discuss the short time part of the ISF.

The RCM assumes that the translational short time dynamics of the trapped water molecule can be treated approximately as the motion of the centre of mass in an isotropic harmonic 
potential well, provided by the mean field of its neighbours. Then, by using the so-called Bloch identity, which is valid for a system with a simple harmonic oscillator Hamiltonian, we connect the short time part of the translational ISF to the centre of mass velocity auto-correlation function, $\left\langle\vec{v}_{\mathrm{CM}}(t) \cdot \vec{v}_{\mathrm{CM}}(0)\right\rangle$ :

$$
F_{\mathrm{T}}^{\mathrm{s}}(Q, t)=\exp \left\{-Q^{2}\left[\int_{0}^{t}(t-\tau)\left\langle\vec{v}_{\mathrm{CM}}(0) \vec{v}_{\mathrm{CM}}(\tau)\right\rangle \mathrm{d} \tau\right]\right\} .
$$

The density of states, $Z(\omega)$, is another key parameter and it has translational and rotational parts. The translational part of the density of states, $Z_{\mathrm{T}}(\omega)$, which can be measured by inelastic neutron scattering experiments, is the Fourier transform of the centre of mass velocity autocorrelation function:

$$
Z_{\mathrm{T}}(\omega)=\frac{1}{2 \pi} \int_{-\infty}^{\infty} \mathrm{e}^{\mathrm{i} \omega t}\left\langle\vec{v}_{\mathrm{CM}}(0) \cdot \vec{v}_{\mathrm{CM}}(\tau)\right\rangle /\left\langle v_{\mathrm{CM}}^{2}\right\rangle \mathrm{d} t
$$

where $M$ is the mass of the particle, and $\left\langle v_{\mathrm{CM}}^{2}\right\rangle=\left\langle v_{x}^{2}\right\rangle+\left\langle v_{y}^{2}\right\rangle+\left\langle v_{z}^{2}\right\rangle=3 v_{0}^{2}=3 k_{\mathrm{B}} T / M$ is the average centre of mass square velocity.

Both MD and experiment results show that the translational harmonic motion of a water molecule in the cage gives rise to two characteristic peaks in $Z_{\mathrm{T}}(\omega)$ (or $Z_{\mathrm{CM}}(\omega)$ ) at about 10 and $30 \mathrm{meV}$, respectively [30]. Therefore, the following Gaussian functional form has been suggested for the translational part of the density of states:

$$
Z_{\mathrm{T}}(\omega)=2(1-C) \frac{\omega^{2}}{\omega_{1}^{2} \sqrt{2 \pi \omega_{1}^{2}}} \exp \left[-\frac{\omega^{2}}{2 \omega_{1}^{2}}\right]+2 C \frac{\omega^{2}}{\omega_{2}^{2} \sqrt{2 \pi \omega_{2}^{2}}} \exp \left[-\frac{\omega^{2}}{2 \omega_{2}^{2}}\right]
$$

where $\sqrt{2} \omega_{1}$ and $\sqrt{2} \omega_{2}$ are the frequencies of the two characteristic peaks in $Z_{\mathrm{T}}(\omega)$, and $C$ is the relative strength of the two peaks. The fit of MD results using equation (10) gives $C=0.44, \omega_{1}=10.8 \mathrm{THz}$, and $\omega_{2}=42.0 \mathrm{THz}$.

We get an expression for $F_{\mathrm{T}}^{\mathrm{s}}(Q, t)$, using equations (8)-(10):

$$
F_{\mathrm{T}}^{\mathrm{s}}(Q, t)=\exp \left\{-Q^{2} v_{0}^{2}\left[\frac{1-C}{\omega_{1}^{2}}\left(1-\mathrm{e}^{-\omega_{1}^{2} t^{2} / 2}\right)+\frac{C}{\omega_{2}^{2}}\left(1-\mathrm{e}^{-\omega_{2}^{2} t^{2} / 2}\right)\right]\right\} .
$$

It should be noted that equation (11) is the short time behaviour of the translational ISF; it starts from unity at $t=0$ and decays rapidly; and in the long time limit (longer than $1 \mathrm{ps),}$ $F_{\mathrm{T}}^{\mathrm{s}}(Q, t)$ decays to a plateau given by an incoherent Debye-Waller factor $A(Q)$ :

$$
A(Q)=\exp \left\{-Q^{2} v_{0}^{2}\left[\frac{1-C}{\omega_{1}^{2}}+\frac{C}{\omega_{2}^{2}}\right]\right\}=\exp \left[-Q^{2} a^{2} / 3\right]
$$

where $a$ is the root-mean-square vibrational amplitude of the water molecules in the cage in which the particle is constrained during its short time movements. Both MD and QENS experiments gave the value $a \approx 0.5 \AA$ in the supercooled region. $a$ is fairly temperature independent [21].

According to the MCT, the cage relaxation at long time can be described by the $\alpha$-relaxation model, with a stretched exponential time decay. This $\alpha$-relaxation model is described by two parameters, the structural relaxation time $\tau_{\mathrm{T}}$, which is $Q$ dependent, and a stretch exponent $\beta$, also slightly $Q$ dependent. Therefore, the complete time dependence of the translational ISF can be written as

$$
F_{\mathrm{T}}(Q, t)=F_{\mathrm{T}}^{\mathrm{s}}(Q, t) \exp \left[-\left(t / \tau_{\mathrm{T}}\right)^{\beta}\right]
$$

Our previous work [23] has shown that $\tau_{\mathrm{T}}$ has a power-law-like dependence on $Q$ with a pre-factor $\tau_{0}$ and a power-law exponent $\gamma$,

$$
\tau_{\mathrm{T}}=\tau_{0}(a Q)^{-\gamma}
$$




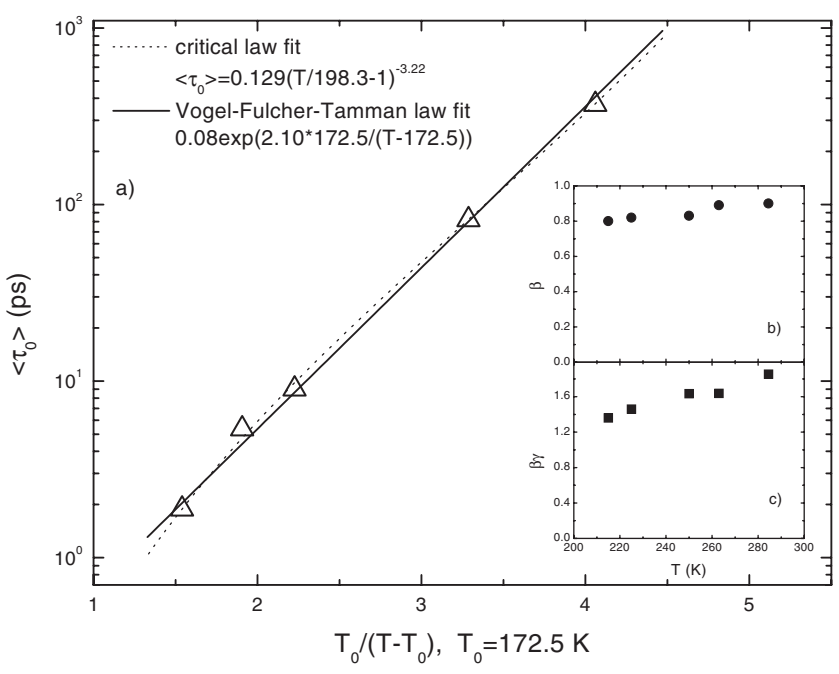

Figure 4. Temperature dependence of the average translational relaxation times as extracted from $\mathrm{SPC} / \mathrm{E}$ water MD data. The inset shows the behaviour of the stretch exponent $\beta$ and of $\beta \gamma$.

We call $\tau_{0}$ the average temperature-dependent translational relaxation time and $\gamma$ the exponent for the $Q$-dependence of $\tau_{\mathrm{T}}$. We therefore focus our discussions on $\tau_{0}$ and $\gamma$ instead of $\tau_{\mathrm{T}}$. MD results show that the exponent $\gamma$ has a slight $Q$-dependence while approaching two in the limit $Q \rightarrow 0$ and remains constant at higher $Q$ values. The stretch exponent, $\beta$, is slightly $Q$ dependent as well while approaching unity at high temperature and in the limit $Q \rightarrow 0$. These limits lead to the normal diffusion process at low $Q$ values, $F_{\mathrm{T}}(Q, t) \approx \exp \left[-D Q^{2} t\right]$, where $D$ is the self-diffusion coefficient. Whereas our experiment is out of this low $Q$ limit, both $\beta$ and $\gamma$ may be considered $Q$ independent $[48,49]$.

In figure 4 we show the temperature dependence of the average translational relaxation time, $\left\langle\tau_{0}\right\rangle=\left(\tau_{0} / \beta\right) \Gamma(1 / \beta)$, as obtained from MD simulations of SPC/E water. The ISFs of the centre of mass at different temperatures, calculated from the MD trajectories, have been fitted according to the RCM, using equations (11) and (13). We fit these results with both the critical law and the Vogel-Fulcher-Tamman law. It is well known that the diffusion coefficient of bulk water follows a critical behaviour [4]. In our case, at low temperature, we cannot extract the diffusion coefficient from the average translational relaxation time, $\left\langle\tau_{0}\right\rangle=\left(\tau_{0} / \beta\right) \Gamma(1 / \beta)$, because it is not $Q^{2}$ dependent. The obtained data follow the critical law: $\left\langle\tau_{0}\right\rangle \sim(T / 198.3-1)^{-3.22}$. This result is in accordance with the reported temperature dependence of the self-diffusion coefficient for SPC/E water [22]: $D \sim(T / 198.7-1)^{2.73}$. Both $D$ and $\left\langle\tau_{0}\right\rangle$ follow a critical law in temperature, with similar $T_{\mathrm{c}}$. Since SPC/E water temperature of maximum density is $T=250 \mathrm{~K}$, there is a $27 \mathrm{~K}$ offset between the real and computer temperature. Therefore, the critical temperature obtained by MD simulations in this case corresponds to $T \approx 225 \mathrm{~K}$ in real water. In the meantime, the Vogel-Fulcher-Tamman (VFT) law shows a good fit to MD generated $\left\langle\tau_{0}\right\rangle$ as well. The VFT law is a way to fit relaxation times close to the glass transition point. According to the VFT law

$$
\tau=\tau_{0} \mathrm{e}^{D T_{0} /\left(T-T_{0}\right)}
$$

where $\tau_{0}, D$, and $T_{0}$ are constants, and $\tau_{0}$ is a vibration time. $T_{0}$ is an ideal glass transition temperature. It is shown in figure 4 that $T_{0}=172.5 \mathrm{~K}$, corresponding to $200 \mathrm{~K}$ in bulk water. It is seen in the inset that $\beta$ is slightly temperature dependent, starting from 0.9 at $284.5 \mathrm{~K}$ 
down to 0.8 at $215 \mathrm{~K} . \beta \gamma$ is the exponent determining the $Q$ dependence of the relaxation time, $\tau_{\mathrm{T}}$. It is seen that at high temperature, the exponent starts from two, signifying a free diffusion, and decreases linearly to a value below two, signifying a restricted diffusion.

\subsection{Theory for the rotational intermediate scattering function}

As far as the rotational ISF is concerned, we start from an exact expansion generated by Sears [54]. As we defined it earlier, $\vec{b}(t)$ denotes a vector from the centre of mass to the hydrogen atom. The rotational ISF could be then expressed by this vector, as the water molecule rotates around the centre of mass,

$$
F_{\mathrm{R}}(Q, t) \equiv\left\langle\mathrm{e}^{-\mathrm{i} \vec{Q} \cdot \vec{b}(0)} \mathrm{e}^{\mathrm{i} \vec{Q} \cdot \vec{b}(t)}\right\rangle=j_{0}^{2}(Q b)+\sum_{\ell=1}^{\infty}(2 \ell+1) j_{\ell}^{2}(Q b) C_{\ell}(t)
$$

where $j_{\ell}(x)$ stands for the $\ell$ th-order spherical Bessel function, $C_{\ell}(t)$ the $\ell$ th-order rotational correlation function, and $b=0.98$ Åthe approximate length of the $\mathrm{O}-\mathrm{H}$ bond in a water molecule. For a typical $Q$ range encountered in QENS experiments, generally $Q<2.5 \AA^{-1}$, this expansion is very useful. The advantage of using this expansion is that the $Q$ dependence of the rotational ISF is exactly given and one needs to make a model for a few lower-order rotational correlation functions which are $Q$-independent quantities. In this case, the expansion needs to be carried out to at most the $\ell=3$ term. In this paper, we shall make a model explicitly for the function $C_{1}(t)$ and shall generate the other higher-order rotational correlation functions approximately using the maximum entropy method of Berne et al [55].

The $\ell$ th-order rotational correlation function is defined as

$$
C_{\ell}(t)=\left\langle P_{\ell}(\cos \theta(t))\right\rangle
$$

where $P_{\ell}$ is the Lengendre polynomial of $\ell$ th order and $\theta(t)$ is the angle between the vector $\vec{b}(0)$ and $\vec{b}(t)$. To calculate the statistical average, we start by considering the short time behaviour of $C_{1}(t)$. At a given instant, say $t=0$, a typical hydrogen atom is hydrogen bonded to its nearest neighbour oxygen atom. Then, the short time dynamics of the rotation of the hydrogen, $\vec{b}(t)$, around the centre of mass must be well described by a harmonic motion of the angle $\theta(t)$, that is to say

$$
\ddot{\theta}(t)+\omega^{2} \theta(t)=0 .
$$

Then, the distribution function of $\theta(t)$ is Gaussian and it follows the Bloch theorem [56]:

$$
\left\langle\mathrm{e}^{\alpha \theta}\right\rangle=\exp \left[\frac{1}{2}\left\langle(\alpha \theta)^{2}\right\rangle\right]
$$

Knowing that

$$
P_{1}(\cos \theta(t))=\cos \theta(t)=\frac{\mathrm{e}^{\mathrm{i} \theta}+\mathrm{e}^{-\mathrm{i} \theta}}{2},
$$

one can then derive the following results:

$$
C_{1}^{\mathrm{s}}(t)=\langle\cos \theta(t)\rangle=\left\langle\frac{\mathrm{e}^{\mathrm{i} \theta}+\mathrm{e}^{-\mathrm{i} \theta}}{2}\right\rangle=\exp \left[-\frac{1}{2}\left\langle\theta^{2}(t)\right\rangle\right] .
$$

Now, since the tip of the vector $\vec{b}(t)$ is tracing a surface of a sphere of radius $b$ centred around the centre of mass, the arc that it traces at short time, $\Delta \vec{b}(t)$, can be considered as a vector in a tangential plane, so one can approximately write $\theta^{2}=\frac{1}{b^{2}}\left(\Delta b_{x}^{2}+\Delta b_{y}^{2}\right)=$ $\left(\int_{0}^{t} \mathrm{~d} t^{\prime} \omega_{x}\left(t^{\prime}\right)\right)^{2}+\left(\int_{0}^{t} \mathrm{~d} t^{\prime} \omega_{y}\left(t^{\prime}\right)\right)^{2}$, where $\vec{\omega}(t)=\frac{1}{b} \frac{\mathrm{d} \vec{b}(t)}{\mathrm{d} t}=\frac{\mathrm{d} \vec{\theta}}{\mathrm{d} t}$ is the angular velocity of the 


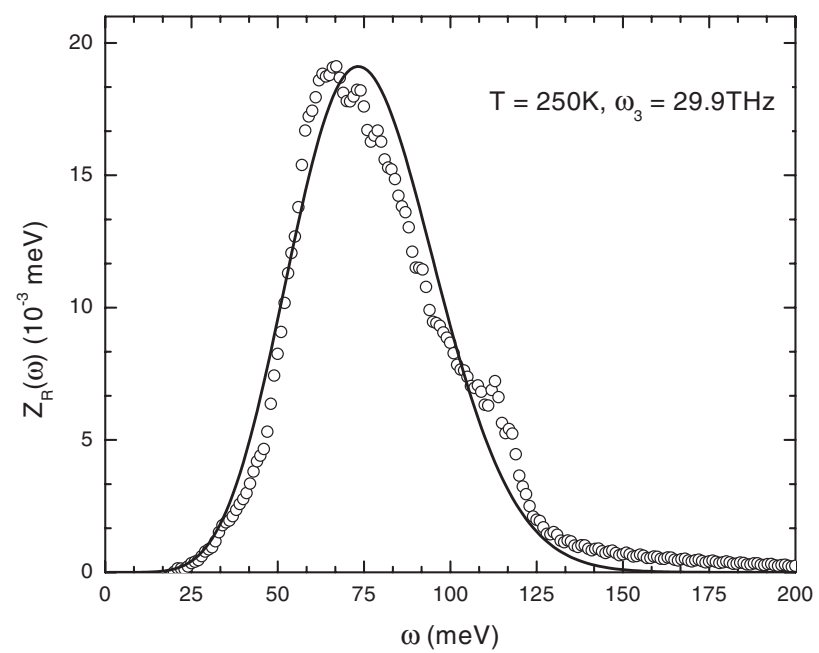

Figure 5. The spectral density function of the normalized angular velocity autocorrelation function $Z_{\mathrm{R}}(\omega)$ at $T=250 \mathrm{~K}$. The open circles represent the results of the simulation and the solid curve the resulting fit by the model using equation (26).

hydrogen atom around the centre of mass. Next, we use the identity

$$
\begin{aligned}
\left\langle\left(\int_{0}^{t} \mathrm{~d} t^{\prime} \omega_{x}\left(t^{\prime}\right)\right)^{2}\right\rangle & =\left\langle\int_{0}^{t} \mathrm{~d} t^{\prime} \int_{0}^{t} \mathrm{~d} t^{\prime \prime} \omega_{x}\left(t^{\prime}\right) \omega_{x}\left(t^{\prime \prime}\right)\right\rangle \\
& =2 \int_{0}^{t}(t-\tau)\left\langle\omega_{x}(0) \omega_{x}(\tau)\right\rangle \mathrm{d} \tau .
\end{aligned}
$$

We finally arrive at a result [57],

$$
\begin{aligned}
C_{1}^{\mathrm{s}}(t) & =\exp \left[-\int_{0}^{t}(t-\tau)\left\langle\omega_{x}(0) \omega_{x}(\tau)+\omega_{y}(0) \omega_{y}(\tau)\right\rangle \mathrm{d} \tau\right] \\
& =\exp \left[-\frac{2}{3} \int_{0}^{t}(t-\tau)\langle\vec{\omega}(0) \cdot \vec{\omega}(\tau)\rangle \mathrm{d} \tau\right]
\end{aligned}
$$

Define the normalized angular velocity auto-correlation function as $\psi_{\mathrm{R}}(t)=\langle\vec{\omega}(0)$. $\vec{\omega}(t)\rangle /\left\langle\omega^{2}\right\rangle$, and its spectral density function as

$$
Z_{\mathrm{R}}(\omega)=\frac{1}{\pi} \int_{-\infty}^{\infty} \mathrm{e}^{\mathrm{i} \omega t} \psi_{\mathrm{R}}(t) \mathrm{d} t
$$

which is normalized to unity for $\omega$ from zero to infinity. Therefore, it is reasonable to make the approximation for the short time part of the first-order rotational correlation function as follows:

$$
C_{1}^{\mathrm{s}}(t)=\exp \left[-\frac{4}{3}\left\langle\omega^{2}\right\rangle \int_{0}^{\infty} \mathrm{d} \omega Z_{\mathrm{R}}(\omega) \frac{1-\cos (\omega t)}{\omega^{2}}\right] .
$$

We model the spectral density function by a simple Gaussian-like function (see figure 5):

$$
Z_{\mathrm{R}}(\omega)=\frac{2 \omega^{6}}{15 \omega_{3}^{6} \sqrt{2 \pi \omega_{3}^{2}}} \exp \left[-\frac{\omega^{2}}{2 \omega_{3}^{2}}\right]
$$

where $\sqrt{6} \omega_{3}$ denotes the peak position. The MD results show that this so-called hindered rotation peak, located approximately at $70 \mathrm{meV}$, is fairly independent of temperature. By 
applying equation (26) to (25), the short time approximation of the first-order rotational correlation function can be written as

$$
\begin{aligned}
C_{1}^{\mathrm{s}}(t) & =\exp \left\{-\frac{2}{3}\left\langle\omega^{2}\right\rangle \int_{0}^{\infty} \mathrm{d} \omega Z_{\mathrm{R}}(\omega) \frac{1-\cos (\omega t)}{\omega^{2}}\right\} \\
& =\exp \left\{-\frac{4\left\langle\omega^{2}\right\rangle}{45 \omega_{3}^{2}}\left[3\left(1-\mathrm{e}^{\frac{-\omega_{3}^{2} t^{2}}{2}}\right)+6 \omega_{3}^{2} t^{2} \mathrm{e}^{\frac{-\omega_{3}^{2} t^{2}}{2}}-\omega_{3}^{4} t^{4} \mathrm{e}^{\frac{-\omega_{3}^{2} t^{2}}{2}}\right]\right\} .
\end{aligned}
$$

This function describes the short time behaviour of the first order rotational correlation function. It starts from unity at $t=0$, exhibits an oscillation at time $0.05 \mathrm{ps}$, and then decays to a flat plateau for times longer than $0.1 \mathrm{ps}$, determined by $\exp \left\{-4\left\langle\omega^{2}\right\rangle / 15 \omega_{3}^{2}\right\}$.

Analogous to the translational dynamics, the first-order rotational correlation function can be separated into a short time harmonic libration in the cage and a long time relaxation of the cage. Therefore, the first-order rotational correlation function can be written as the product of a short time libration and a long time stretched exponential relaxation:

$$
C_{1}(t)=C_{1}^{\mathrm{s}}(t) \exp \left[-\left(t / \tau_{\mathrm{R}}\right)^{\beta}\right]
$$

where $\tau_{\mathrm{R}}$ is the rotational relaxation time and $\beta$ is the stretch exponent, the same as in $F_{\mathrm{T}}(Q, t)$, and the reason will be described later.

The whole picture resembles the relaxing cage model of the translational dynamics. At short time, the orientation of the central water molecule is fixed by the $\mathrm{H}$ bonds with its neighbours. It performs nearly harmonic oscillations around the hydrogen-bond direction, described by $C_{1}^{\mathrm{s}}(t)$. At longer times, the bonds break and the cage begins to relax, so that the particle can reorient itself, losing memory of its initial orientation. Thus, the first-order rotational correlation function eventually decays to zero by a stretched exponential relaxation.

We define a notation, $\mu(t)=\cos \theta(t)$. To calculate $C_{2}(t)$ and $C_{3}(t)$ from $C_{1}(t)$, we need a probability distribution function, $P(\mu, t)$, and we need to know its functional form. We shall guess the distribution function based on maximization of the informational entropy subject to the condition that we know $C_{1}(t)$ [55]. This method is very effective at short times, corresponding to the harmonic libration. According to the scheme, the distribution function is given by

$$
P(\mu, t)=\mathrm{e}^{\alpha+\beta \mu}
$$

Because $\int \mathrm{d} \Omega P(\mu, t)=1$,

$$
\begin{aligned}
& \mathrm{e}^{\alpha}=\frac{1}{2 \pi} \frac{\beta}{\mathrm{e}^{\beta}-\mathrm{e}^{-\beta}}, \\
& C_{1}(t)=\int \mathrm{d} \Omega \mathrm{e}^{\alpha+\beta \mu} \mu=-[1 / \beta(t)]+\operatorname{coth} \beta(t) .
\end{aligned}
$$

Then the higher-order correlation functions can be determined from $C_{1}(t)$ using equations (29)-(31). The connection of $C_{1}(t)$ to the higher-order rotational correlation functions is given in terms of $\beta(t)$. The results are

$$
\begin{aligned}
& C_{2}(t)=1-[3 / \beta(t)] C_{1}(t), \\
& C_{3}(t)=-\frac{5}{\beta(t)}+\left[1+\frac{15}{\beta(t)}\right] C_{1}(t) .
\end{aligned}
$$

\subsection{The validity of the theory of the rotational correlation functions}

To explain the validity of the theory of the rotational correlation function, we focus on the validity of $C_{1}(t)$ as given by equations (25) and (28). Since the short time behaviour of the firstorder rotational correlation function, $C_{1}^{\mathrm{s}}(t)$, is essentially determined by the spectral density 
function of the normalized angular velocity autocorrelation function, $Z_{\mathrm{R}}(\omega)$ (equation (25)), we show in figure 5 the MD data of $Z_{R}(\omega)$ and its representation by the analytical function, $\left[2 \omega^{6} /\left(15 \omega_{3}^{6} \sqrt{2 \pi \omega_{3}^{2}}\right)\right] \exp \left[-\omega^{2} /\left(2 \omega_{3}^{2}\right)\right]$. It is obvious that a broad band is peaked at $\sim 65 \mathrm{meV}$ for the MD data. In the Gaussian representation by equation (26), the peak position is at $\sqrt{6} \omega_{3}$. We note that this analytical function is a fair representation of the spectral density function.

Next, we show that $Z_{R}(\omega)$ is part of the spectral density function of the hydrogen atom. Since we know that

$$
\vec{v}_{\mathrm{H}}(t)=\vec{v}_{\mathrm{CM}}(t)+\vec{v}_{\mathrm{R}}(t)
$$

and

$$
\vec{v}_{\mathrm{R}}(t)=b \vec{\omega}(t)
$$

we get the relation

$$
\left\langle\vec{v}_{\mathrm{H}}(0) \cdot \vec{v}_{\mathrm{H}}(t)\right\rangle=\left\langle\vec{v}_{\mathrm{CM}}(0) \cdot \vec{v}_{\mathrm{CM}}(t)\right\rangle+b^{2}\langle\vec{\omega}(0) \cdot \vec{\omega}(t)\rangle
$$

in which we neglect the cross terms because they are very small compared with the others at short time. Moreover, since

$$
\begin{aligned}
& Z_{\mathrm{H}}(\omega)=\frac{1}{\pi} \int_{-\infty}^{\infty} \mathrm{e}^{\mathrm{i} \omega t} \frac{\left\langle\vec{v}_{\mathrm{H}}(0) \cdot \vec{v}_{\mathrm{H}}(t)\right\rangle}{\left\langle v_{\mathrm{H}}^{2}\right\rangle} \mathrm{d} t, \\
& Z_{\mathrm{CM}}(\omega)=\frac{1}{\pi} \int_{-\infty}^{\infty} \mathrm{e}^{\mathrm{i} \omega t} \frac{\left\langle\vec{v}_{\mathrm{CM}}(0) \cdot \vec{v}_{\mathrm{CM}}(t)\right\rangle}{\left\langle v_{\mathrm{CM}}^{2}\right\rangle} \mathrm{d} t
\end{aligned}
$$

one can safely write

$$
Z_{\mathrm{H}}(\omega) \simeq \alpha Z_{\mathrm{CM}}(\omega)+\beta Z_{\mathrm{R}}(\omega)
$$

where

$$
\alpha+\beta=1 .
$$

In figure 6 we plot MD results for $Z_{\mathrm{H}}(\omega)$ and its decomposition into the sum of $Z_{\mathrm{CM}}(\omega)$ and $Z_{\mathrm{R}}(\omega)$ for $T=225 \mathrm{~K}$. From the inspection of the figure, it is obvious that the two low frequency peaks of the hydrogen density of states are translational in character and the prominent high frequency peak belongs to rotations. In the literature, the latter peak is often called the hindered rotation peak, which is clearly associated with the oscillation of the hydrogen atom perpendicular to its hydrogen bond.

After applying the theory for $C_{1}^{\mathrm{s}}(t)$ to $C_{1}(t)$ and deriving $C_{2}(t)$ and $C_{3}(t)$ through $C_{1}(t)$, we are now ready to compute the rotational ISF using Sears expansion (equation (16)). Figure 7 shows the rotational ISF calculated by MD at three $Q$ values and their computation by Sears expansion using the MD generated $C_{1}(t), C_{2}(t)$, and $C_{3}(t)$. For all three $Q$ values, one sees good agreements between the two, indicating that up to $Q=2.26 \AA^{-1}$ our theoretical model for the rotational correlation functions is valid and the Sears expansion can be safely truncated at the fourth term.

To see the critical behaviour of rotational dynamics, we have analysed SPC/E water MD simulation trajectories to obtain $C_{1}(t)$ and $C_{2}(t)$. Their long time relaxation has been fitted to a stretched exponential function. Figure 8 shows the obtained results. The data obey a critical law with $T_{\mathrm{c}}^{\mathrm{MD}}=204 \mathrm{~K}$, which is close to the critical temperature found for the translational dynamics of SPC/E water. The data obey the VFT law as well with $T_{0}\left(\left\langle\tau_{\mathrm{R}_{1}}\right\rangle\right)=160.4 \mathrm{~K}$ and $T_{0}\left(\left\langle\tau_{\mathrm{R}_{2}}\right\rangle\right)=170.5 \mathrm{~K}$, which is near the value obtained from the average translational relaxation times, $T_{0}=172.5 \mathrm{~K}$, signalling an ideal glass transition at about $200 \mathrm{~K}$ (real temperature).

We show the $\beta$ values for the fitting of $C_{1}(t)$ and $C_{2}(t)$, in figure 8 inset (b). At the same time, the $\beta$ values for the translational dynamics are shown denoted as $\beta$. Within the error limit, they can be considered coincident, which confirms the validity of the assumption, in the fitting algorithm, that $\beta_{\mathrm{R}}=\beta$. 




Figure 6. The spectral density function of the normalized velocity autocorrelation function of the hydrogen atoms, $Z_{\mathrm{H}}(\omega)$, and its decomposition into the weighted sum of $Z_{\mathrm{R}}(\omega)$ and $Z_{\mathrm{CM}}(\omega)$, where the latter quantity represents the spectral density function of the normalized centre of mass velocity autocorrelation function. It is to be noted that the prominent peak at $65 \mathrm{meV}$, the so-called hindered rotation peak, hardly shifts as a function of temperature.

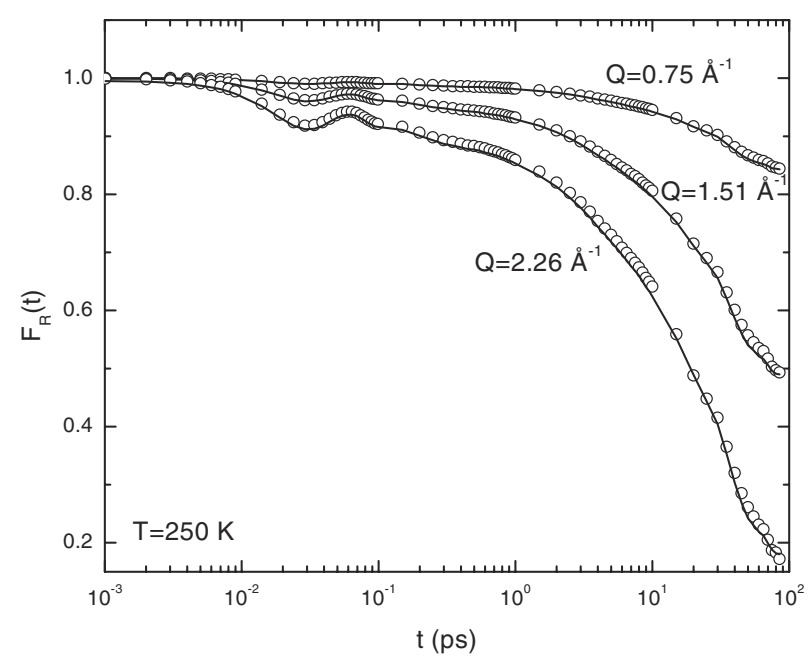

Figure 7. The rotational intermediate scattering function $F_{\mathrm{R}}(Q, t)$ versus time at three $Q$ values and at $T=250 \mathrm{~K}$. From top to bottom, $Q=0.75,1.51$, and $2.26 \AA^{-1}$. The open circles represent simulated $F_{\mathrm{R}}(Q, t)$ at each $Q$ value, the solid curves the results computed by Sears expansion equation (16) up to the fourth-order term using simulated $C_{1}(t), C_{2}(t)$, and $C_{3}(t)$.

\subsection{The coupling of the translational and rotational dynamics}

It has been clearly shown, using MD simulation, that translational and rotational dynamics are strongly coupled [24]. In fact, the long time behaviour of the first, second, and thirdorder rotational correlation functions, $C_{1}(t), C_{2}(t)$, and $C_{3}(t)$, coincides with the long time behaviour of the ISF of the centre of mass at three specific $Q$ values, $Q_{1}^{*}, Q_{2}^{*}$, and $Q_{3}^{*}$, independent of temperature $[49,58]$. Even though the model uses the decoupling 


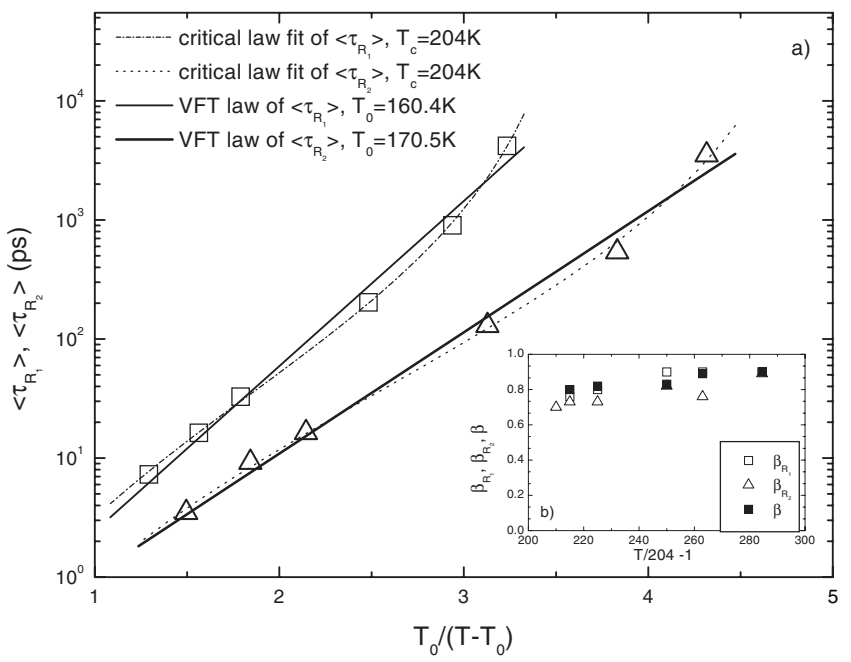

Figure 8. Temperature dependence of the average relaxation times for the first- and second-order rotational correlation functions as extracted from SPC/E water MD data. The inset shows the stretch exponents.



Figure 9. MD results for the temperature dependence of the parameter $Q^{*}$.

approximation, which neglects translational-rotational correlations, we impose the same stretch exponent, $\beta$, for both the translational ISF and the first-order rotational correlation function. Therefore, at a specific $Q^{*}$, the long time decay of $F_{\mathrm{T}}\left(Q^{*}, t\right)$ and $C_{1}(t)$ coincide: $\exp \left[-\left(t / \tau_{\mathrm{T}}\left(Q^{*}\right)\right)^{\beta}\right]=\exp \left[-\left(t / \tau_{\mathrm{R}}\right)^{\beta}\right]$, where $Q^{*}$ is given by

$$
Q^{*}=\frac{1}{a}\left(\frac{\tau_{0}}{\tau_{\mathrm{R}}}\right)^{1 / \gamma} \text {. }
$$

Figure 9 is the MD generated $Q^{*}$. It is between 1.0 and 1.2 at all temperatures, which could be seen as temperature independent. 




Figure 10. Microscopic structures of MCM-41-S (pcb-159c) and MCM-48-S (pcb-072c).

\section{Experiment}

\subsection{Preparation and characterization of the samples}

We use two samples, MCM-41-S (pcb-159-c, with pore size $25 \AA$ ) and MCM-48-S (pcb-072c, with pore size $22 \AA$ ) to extract most of our results and compare with RCM predictions and MD simulation results. Two other samples, MCM-41-S (mac-1219-7, with pore size $18 \AA$, and mac-0124-4, with pore size $14 \AA$ ) have also been tested and are shown in a few figures. Figure 10 shows the schematic microstructures of MCM-41-S (pcb-159-c) and MCM-48-S (pcb-072c). The former matrix (25 $\AA$ pore diameter) has 1D cylindrical tubes arranged in a hexagonal structure whereas the latter (22 $\AA$ pore diameter) has a 3D bicontinous morphology. We explain the preparation and characterization of these samples in the following. To synthesize the MCM samples, zeolite seeds were prepared by mixing $\mathrm{NaAlO}_{2}$ (Riedel-de Haen), $\mathrm{NaOH}$ (Shimakyu's Pure Chemicals, Japan), fumed silica (Sigma), tetraethyl ammonium hydroxide (TEAOH) aqueous solution (20\%) (Acros), and water with stirring for $2-5 \mathrm{~h}$, then transferring the solution into an autoclave at $100{ }^{\circ} \mathrm{C}$ for $18 \mathrm{~h}$. A clear solution of nanoprecursors was obtained. MCM-48-S (pcb-072c) [59] was synthesized by reacting zeolite seeds with cetyltrimethylammonium bromide solution (CTAB, Acros) at $150{ }^{\circ} \mathrm{C}$ for $6-24 \mathrm{~h}$. Samples were then collected by filtration, washed with water, dried at $100^{\circ} \mathrm{C}$ in an oven for $6 \mathrm{~h}$, and calcined at $580^{\circ} \mathrm{C}$ for $6 \mathrm{~h}$. To obtain MCM-41-S (pcb-159c) [60] the mixture of zeolite seeds and surfactant solution was hydrothermally treated at $150{ }^{\circ} \mathrm{C}$ for 2-6 h, creating a disordered mesostructure. After $\mathrm{pH}$ adjustment and hydrothermal reaction at $100{ }^{\circ} \mathrm{C}$ for 2 days we obtained the desired hexagonal nanoporous material. The molar ratios of the reactants $\mathrm{NaAlO}_{2}: \mathrm{SiO}_{2}: \mathrm{NaOH}: \mathrm{TEAOH}: \mathrm{C}_{16}$ TMAB: $\mathrm{H}_{2} \mathrm{O}$ were 1:37-67:1.5-9:1122:18.3:3000-3500 for both cases.

The samples have been characterized using X-ray diffraction (XRD) (Scintag X1 diffractometer using $\mathrm{Cu} \mathrm{K} \alpha, \lambda=0.154 \mathrm{~nm}$ ), transmission electron microscopy (TEM), thermogravimetric analysis (TGA) (NETZSCH TG-209), and differential scanning calorimetry (DSC) (TA Instrument 5100 control system and LT-Modulate DSC 2920). $\mathrm{N}_{2}$ adsorption-desorption isotherms have been measured as well (Micromeritics ASAP 2010 sorptometer at $77 \mathrm{~K}$ ).

XRD spectra show that the MCM-48-S was well ordered with cubic ( $I a 3 d)$ symmetry, whereas the MCM-41-S had hexagonal $(P 6 \mathrm{~mm})$ symmetry, as shown in figure 11. Both samples exhibit high hydrothermal stability. BET surface area, pore size, and pore volume were determined using the nitrogen adsorption-desorption technique. The obtained values are 

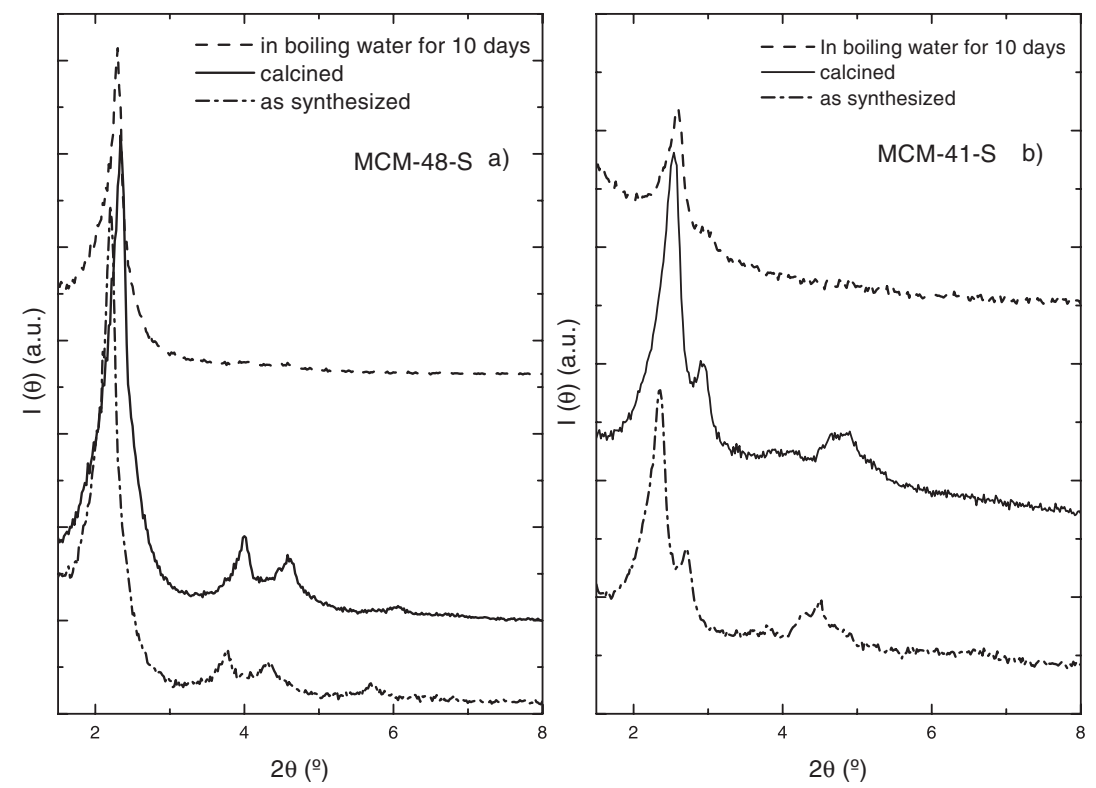

Figure 11. XRD patterns of MCM-48-S (pcb-072c) and MCM-41-S (pcb-159c).

Table 2. Parameters characterizing the structural properties of the investigated samples.

\begin{tabular}{llll}
\hline & Surface area $\left(\mathrm{m}^{2} \mathrm{~g}^{-1}\right)$ & Pore size $(\AA)$ & Pore volume $\left(\mathrm{cm}^{3} \mathrm{~g}^{-1}\right)$ \\
\hline MCM-48-S & 1312 & 22.0 & 0.97 \\
MCM-41-S & 1155 & 25.0 & 1.11 \\
\hline
\end{tabular}

reported in table 2. The wall thickness of the MCM-48-S sample is about $10 \AA$, as calculated using the Schumacher method [61].

To hydrate the nanoporous matrices, we put the calcined samples onto the diskette that was full of water $\left(\mathrm{H}_{2} \mathrm{O}\right)$ vapour by pumping with saturated $\mathrm{NaCl}$ solution for 2-3 days. We thus obtained water-loaded samples with hydration levels $\left(\mathrm{gr}_{\mathrm{H}_{2} \mathrm{O}} / \mathrm{gr}_{\text {sample }}\right)$ of $\approx 30 \%$ (MCM$41-\mathrm{S}$ ) and $\approx 35 \%$ (MCM-48-S), as determined by thermogravimetric analysis. Even though the MCM-41-S sample has a larger pore volume, it has a lower hydration level. This discrepancy is probably connected to the presence of void defects.

According to the Gibbs-Thomson equation, the melting point of a small crystal is inversely proportional to the crystal size, which, in this case, is equal to the pore size of the material. The melting/freezing behaviour of water in the samples has been checked by DSC measurements (figure 12). Thus, the MCM-41-S sample (pore size $25 \AA$ ) has a higher melting point than the MCM-48-S sample (pore size $22 \AA$ ). The estimated melting points are $T_{\mathrm{m}}=229.5$ and $232.4 \mathrm{~K}$, for water in the MCM-48-S and MCM-41-S matrices, respectively. Using a backscattering spectrometer, we have also recorded a series of elastic scans. In figure 13 we report, as an example, the elastic scan for the MCM-48-S (22 $⿱$ ) and MCM-41-S (14 $\AA$ ) hydrated samples. We took down the fraction of neutrons elastically scattered by the samples, in the temperature range from $T=150$ to $300 \mathrm{~K}$, with an energy resolution of $1 \mu \mathrm{eV}$. As we decrease the temperature, the fraction of elastic scattering increases. In fact, the dynamics of the samples slows down and more and more neutrons are detected at the elastic channel. For the $22 \AA$ sample, at about $T=220 \mathrm{~K}$ a plateau is reached, whereas for the $14 \AA$ sample a plateau 


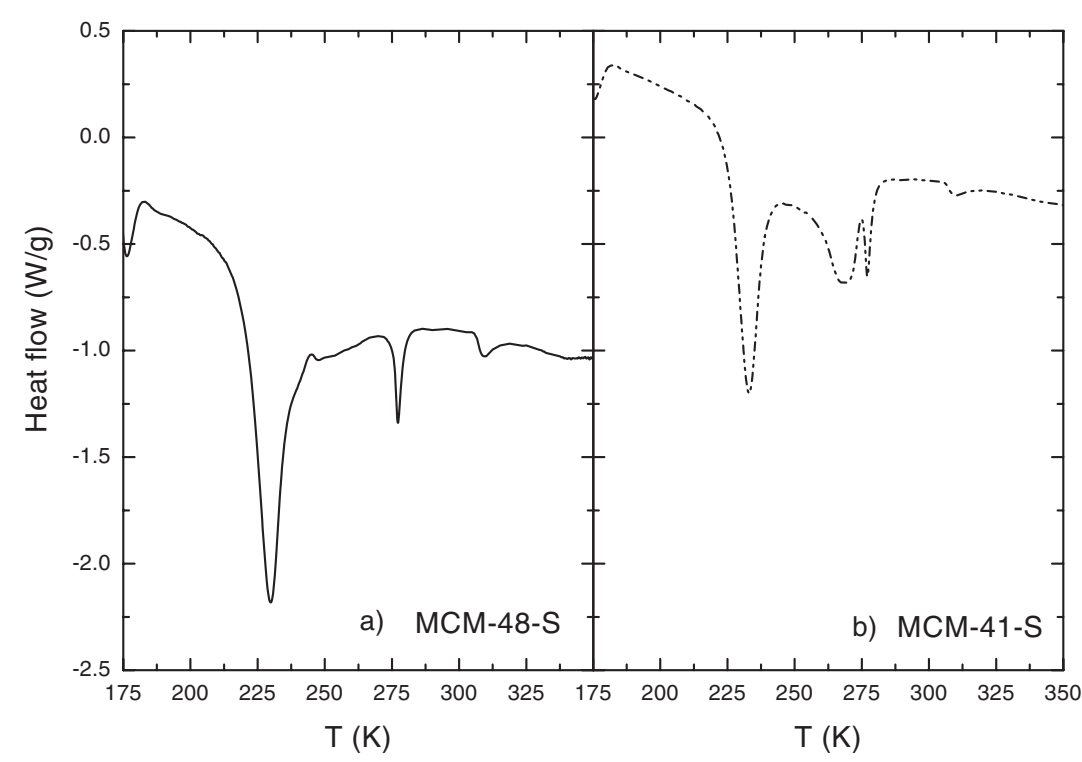

Figure 12. Differential scanning calorimetry curves of water inside MCM-48-S (pore size $22 \AA$, panel (a)) and MCM-41-S (pore size $25 \AA$, panel (b)) samples.

is reached at about $T=200 \mathrm{~K}$, signalling that the dynamics freezes at these temperatures. The values obtained using neutron scattering are in good agreement with the DSC results.

\subsection{QENS experiments}

In the QENS experiments, the hydrated powder of MCM-41-S and MCM-48-S was evenly spread to form a rectangular slab sample $0.5 \mathrm{~mm}$ thick, such that multiple-scattering corrections should not be necessary (transmission $95 \%$ ).

We carried out the QENS measurements at the NIST Centre for Neutron Research, using the Fermi chopper (FCS), the disc chopper (DCS), and the backscattering (HFBS) spectrometers. For FCS and DCS the incident neutron wavelength was $6.0 \AA$ and the Gaussian energy resolution function had a full width at half maximum (FWHM) of $\sim 60$ and $\sim 30 \mu \mathrm{eV}$ respectively. For HFBS we have chosen the low resolution configuration, corresponding to a resolution function with FWHM of $\sim 1.0 \mu \mathrm{eV}$ and an energy window of $\pm 36 \mu \mathrm{eV}$. In all cases, the rectangular sample cell was placed at $45^{\circ}$ to the direction of the incident neutron beam. The detectors facing the edge of the can have been discarded. The resulting range of elastic wavevector $Q$ was from 0.33 to $1.93 \AA^{-1}$, from 0.27 to $1.93 \AA^{-1}$, and from 0.25 to $1.60 \AA^{-1}$, in the case of FCS, DCS, and HFBS respectively. These $Q$ ranges are broad enough to simultaneously monitor both translational and rotational dynamics of the water molecules. The spectra were corrected for scattering from the same sample holder, standardized using results from a run using vanadium and converted to the differential scattering cross section using standard routines available at NIST.

\section{Results and discussion}

\subsection{General fit}

Figure 14 is an example of comparison of a recorded experimental spectrum with the fitted result using the RCM model. The data, collected using FCS, refer to a sample of MCM-48-S 


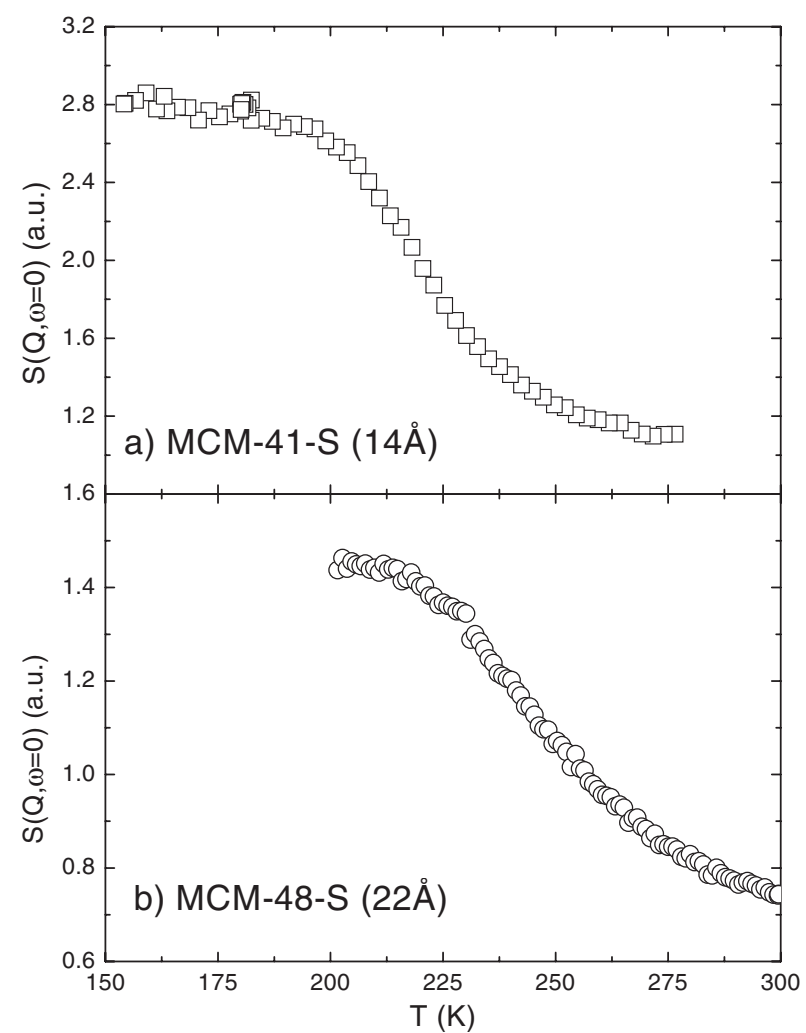

Figure 13. The elastic scans of the MCM-48-S (pore size $22 \AA$ ) and MCM-41-S (pore size $14 \AA$ ) samples.

at $T=300 \mathrm{~K}$, at $Q=1.88 \AA^{-1}$. It is seen that the agreement between the model and the experimental data is satisfactory throughout the entire spectral range.

As shown in figure 14, a pronounced elastic component is superimposed on the quasielastic broadening. The presence of this quasielastic component is clearly detectable at most temperatures and $Q$, even with the broad resolution of FCS. The elastic contribution is mainly due to the scattering of hydrogen atoms in surface silanol $(\mathrm{Si}-\mathrm{O}-\mathrm{H})$ groups [28]. The dangling $\mathrm{O}$ and $\mathrm{H}$ atoms of water molecules are connected to the surface $\mathrm{Si}$ atom to form a silanol group. In this silanol group, the $\mathrm{H}$ atom is not fixed, but is constrained to move in a circle in the surface to keep the $\mathrm{O}-\mathrm{H}$ bond length and the $\mathrm{Si}-\mathrm{O}-\mathrm{H}$ angle constant. It is known that, for the hydroxylated silica surface, surface solvent hydrogen bonding is stronger than interactions in the bulk solvent, with the nearest solvent layer interacting specifically with up to three surface hydroxyl groups. Therefore, the strong interactions between water molecules and the silanol groups should be taken into account in our analysis as well.

As temperature decreases, water dynamics slows down very fast and it is increasingly difficult for it to be resolved by the finite resolution of QENS spectrometers. Some of the water molecules are so slow that they could be seen as immobile by the QENS spectrometers and contributing to the elastic component, so that the elastic contribution increases as temperature decreases. It has been also found that the dynamics of water in nanoporous materials at supercooled temperature is better described by a distribution of relaxation times than a single average relaxation time. 


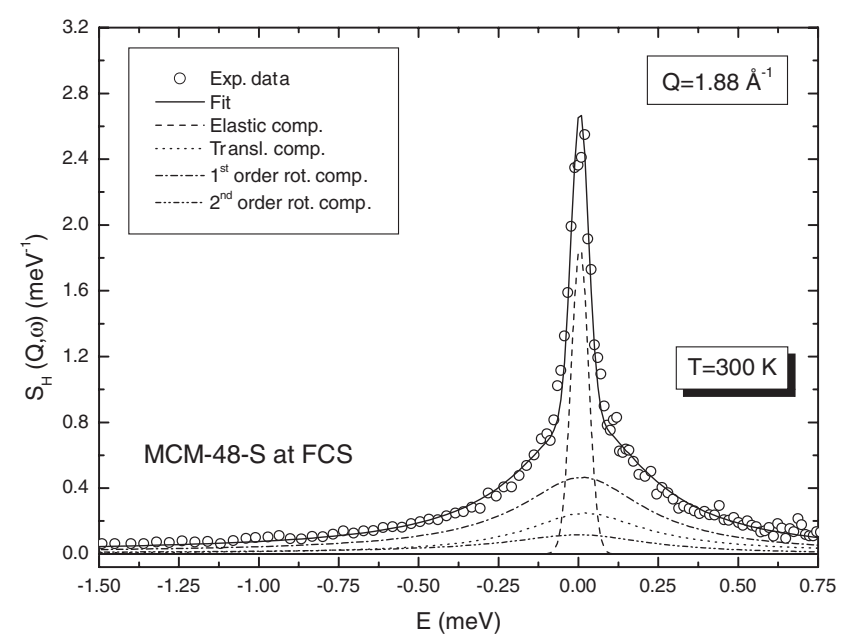

Figure 14. A typical QENS spectra from the MCM-48-S hydrated sample at $T=300 \mathrm{~K}$, at $Q=1.88 \AA^{-1}$. Data have been collected using FCS. Error-bars are not shown for the sake of the clarity of the figure; the scatter of the data furnishes an idea of the error. The curves show the different components of the data analysis: the continuous curve represents the result of the fit; the dashed curve is the elastic component; the dotted, dash-dot, and dash-dot-dot curves represent contributions to the scattering from the first three terms of the Sears expansion, respectively.

Defining the magnitude of the wavevector transfer $Q$ corresponding to the scattering angle $\theta$ at the elastic channel as $Q_{0}=[4 \pi \sin (\theta / 2)] / \lambda$, we can analyse the experimental data according to the following equation:

$$
S(Q, \omega)=p R\left(Q_{0}, \omega\right)+(1-p) F T\left\{F_{\mathrm{H}}(Q, t) R\left(Q_{0}, t\right)\right\}
$$

where $p$ is the area of the elastic component, $F_{\mathrm{H}}(Q, t)$ is the ISF of hydrogen atoms, and $R\left(Q_{0}, t\right)$ is the Fourier transform of the experimental resolution function, $R\left(Q_{0}, \omega\right)$.

Taking the weight of the elastic component as a fitting parameter, we obtain temperaturedependent values for $p$. However, in this way we were trying to evaluate contributions from the hydrogen atoms of surface silanol groups, the water molecules interacting strongly with surface silanol groups, and some of the very slow bulk water molecules. At temperature values lower than $270 \mathrm{~K}$, the area of the elastic component seems to increase, whereas for $T \geqslant 270 \mathrm{~K}$ a plateau is reached. The fast increase on lowering temperature is obviously connected to the slowing down of water dynamics. In the meantime, the plateau value is then the scattering due to the hydrogen atoms of surface silanol groups, without the contribution of bulk water and the water molecules interacting strongly with surface silanol groups. The surface silanol groups are so well organized and strong that they may be seen as temperature independent. We can find as well that even if FCS and DCS results agree very well, a sudden jump occurs when the data from HFBS are considered as well. This occurrence is due to the different resolution function, more than one order of magnitude sharper than for DCS. In the ideal case, the area of the elastic component would be resolution independent, if two clearly separated relaxation times can be identified with one inside the experimental time window and the other corresponding to virtually immobile particles. However, experimentally, we expect a continuous distribution of relaxation times in supercooled water, encompassing the dynamics of water in the inner part and on the surface of the pores. Therefore, the value of $p$ is strongly dependent on the time-window cut-off. For this reason, to obtain consistent results, we have fixed $p$ to its 


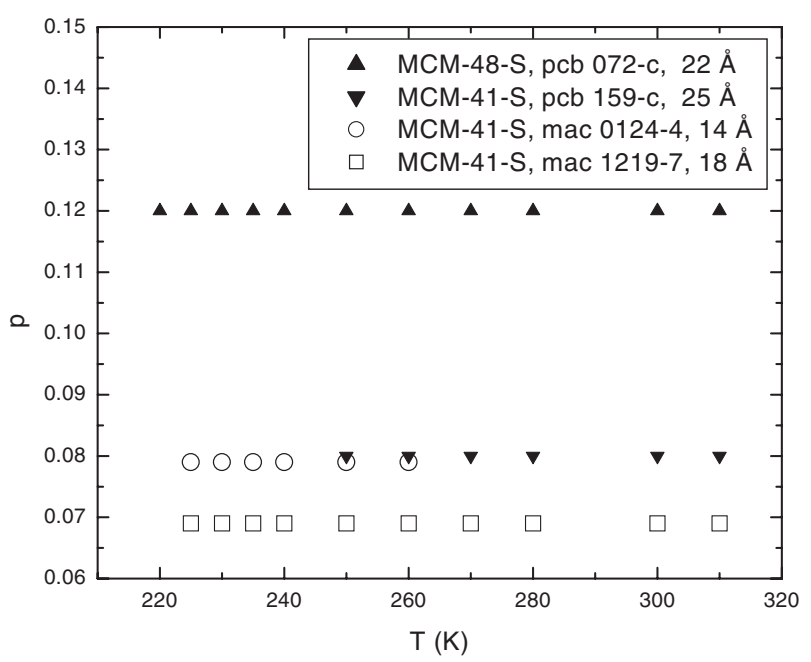

Figure 15. The elastic component weight, $p$, for the four samples as measured using the three QENS spectrometers: FCS, DCS, and HFBS.

plateau value, $p=0.12$ and 0.08 in the case of MCM-48-S and MCM-41-S, respectively, when analysing the data from different spectrometers (see figure 15). We argue that, without introducing an arbitrary cut-off, it is impossible to identify a population of molecules whose dynamics is much slower than the resolution of the instrument. More consistent results from different instruments can then be obtained by assuming a single distribution of relaxation times for the whole water population. It would be expected that $p$ of MCM-48-S should be smaller than that of MCM-41-S since the water content in the MCM-48-S sample is slightly higher than in the MCM-41-S case. However, the fixed plateau value for MCM-48-S is slightly higher than MCM-41-S, because the number of silanol groups is larger in the sample with larger surface area (MCM-48-S).

The quasielastic broadening has been analysed according to the RCM as described in the previous section. $F_{\mathrm{H}}(Q, t)$ is described in terms of ten parameters (from equations (4), (5), (11), (13), (16), (27), (28), (32), (33) and (41)): $C, \omega_{1}, \omega_{2}, \tau_{0}, \gamma, \beta, \omega_{3}, \tau_{\mathrm{R}},\left\langle\omega^{2}\right\rangle$, and $p$. Five of them are related to the short time dynamics, namely $C, \omega_{1}, \omega_{2}, \omega_{3}$, and $\left\langle\omega^{2}\right\rangle$. The short time dynamics is not strongly temperature dependent, according to MD simulation results. On the other hand, the quasielastic broadening is mostly determined by the long time dynamics. For this reason, we have fixed the values of $C, \omega_{1}, \omega_{2}$, and $\omega_{3}$ according to the results of our MD simulations. This procedure is reliable. In fact, MD simulation results on the short time dynamics can be compared with the experimental results from inelastic neutron scattering, and the agreement is very satisfactory $[23,24] .\left\langle\omega^{2}\right\rangle$ has been fixed according to the equation $\left\langle\omega^{2}\right\rangle=k_{\mathrm{B}} T / I$, through an estimation of the momentum of inertia of the water molecules. This value is in agreement with the results of MD simulations [24] and of the combined analysis of QENS and INS spectra [50].

The remaining parameters, namely $p, \tau_{0}, \gamma, \tau_{\mathrm{R}}$, and $\beta$, can be determined from the analysis of the QENS spectra. However, in the remainder of the paper, we report the results of our analysis with the value of $p$ fixed to its plateau value. Therefore, since the fitting parameters are $Q$ independent, we fit the experimental $S(Q, \omega)$ surface (both $\omega$ and $Q$ dependence) using just four fitting parameters. We took into account the first three terms of the Sears expansion. We actually fitted constant angle spectra, taking into account the dependence of $Q$ on the 




Figure 16. Comparison of the typical QENS spectra of the MCM-48-S sample from the three different spectrometers, at three different temperatures. The continuous, dash, dot, dash-dot, and dash-dot-dot curves represent the total fit, the elastic component and the contributions from the first three terms of the Sears expansion, respectively.

energy transfer, $E$ :

$$
Q=\sqrt{2 m_{\mathrm{n}}\left[2 E_{\mathrm{i}}-E-2 \cos \theta \sqrt{E_{\mathrm{i}}\left(E_{\mathrm{i}}-E\right)}\right]}
$$

where $m_{\mathrm{n}}$ is the mass of the neutron and $E_{\mathrm{i}}$ is the energy of the incident neutrons.

In figure 16, we show as an example three spectra collected using FCS, DCS, and HFBS. From the figure, the progressive sharpening of the quasielastic broadening as temperature is lowered can be noticed. The $1 \mu \mathrm{eV}$ resolution of HFBS is necessary to obtain useful data at $T \leqslant 250 \mathrm{~K}$, even if the resulting energy window is quite small. As the broadening is strongly temperature dependent, the data have been fitted in different energy windows for the three spectrometers. FCS and DCS data have been analysed in the ranges $-5 \mathrm{meV} \leqslant E \leqslant 0.8 \mathrm{meV}$ and $-2 \mathrm{meV} \leqslant E \leqslant 0.9 \mathrm{meV}$, respectively. Such big ranges are necessary to the analysis of the rotational components, whose wings are very large at the high $Q$ values. In any spectra, no appreciable background has been noticed. The HFBS energy window is from -30 to $30 \mu \mathrm{eV}$. In this small interval, the $Q$ value can be considered equal to the zero-energy-transfer value, $Q_{0}$, in the whole range. 




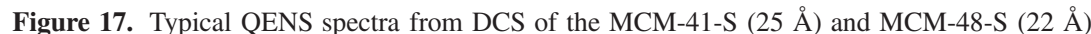
sample at $T=260 \mathrm{~K}$, at three different $Q$ values, $0.63,1.41$, and $1.93 \AA^{-1}$. The errors are no larger than the symbols. The continuous curve represents the result of the fit; the dashed curve is the elastic component; the dotted, dash-dot, and dash-dot-dot curves represent contributions to the scattering from the first three terms of the Sears expansion, respectively.

Figures 17 and 18 show the $Q$ dependence of the spectra for MCM-48-S (22 $\AA$ ) and MCM-41-S (25 $\AA$ ) using the three spectrometers. The scatter of the data furnishes an idea of the error. The fitting results are shown as well, as continuous lines. In figures 14 and 16-18, the first three terms of the Sears expansion, used in the analysis procedure, are reported as dot, dash-dot, and dash-dot-dot curves. As can be seen, the fit is very good in all investigated cases. It is remarkable that using four parameters we were able to reproduce the data from 11 (FCS), 9 (DCS), and 7 (HFBS) constant angle spectra.

As mentioned in the previous section in all cases, we have analysed, at the same time, a low angle reflection geometry and a high angle transmission geometry group of spectra. 


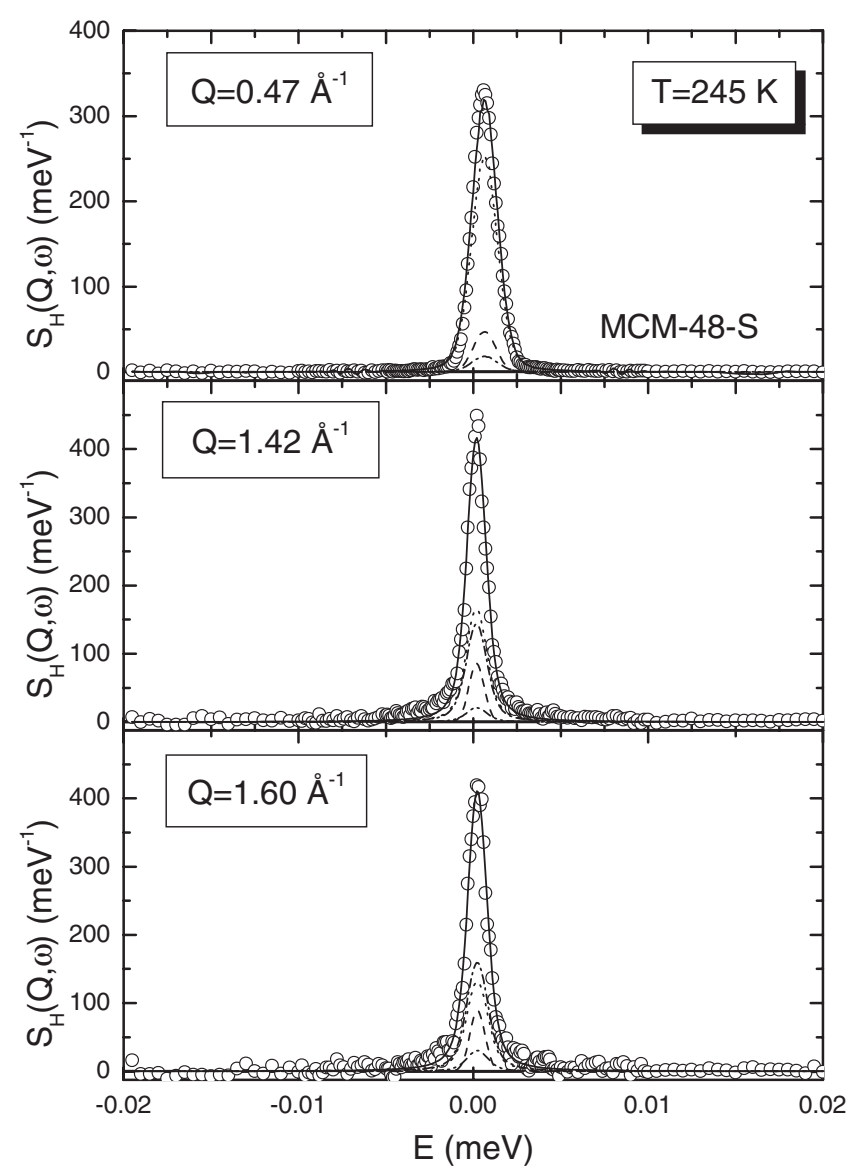

Figure 18. Typical QENS spectra from HFBS of the MCM-48-S sample at $T=245 \mathrm{~K}$, at three different $Q$ values, $0.47,1.42$, and $1.60 \AA^{-1}$. The scatter of the data furnishes an idea of the error. The continuous curve represents the result of the fit; the dashed curve is the elastic component; the dotted, dash-dot, and dash-dot-dot curves represent contributions to the scattering from the first three terms of the Sears expansion, respectively.

Although the lowest angle spectra are somewhat resolution limited, from the first group, the translational dynamics can be obtained. As $Q$ increases, the spectra broaden, both because of the $Q$ dependence of the translational dynamics and because of the increasing contribution from the rotational dynamics at $Q \geqslant 1 \AA^{-1}$. Therefore, the high $Q$ spectra are determined by the translational and rotational dynamics. The simultaneous fitting of the spectra at different angles allows the separation of the different contributions.

In tables 3-5 we report the extracted fitting parameters for the FCS, DCS, and HFBS data respectively. In the tables the $\chi^{2}$ of the total fit is reported as well. As can be seen it increases with decreasing temperature, possibly because, as the dynamics slows down, the finite resolution of the instrument does not allow a perfect fit. It can also be seen that, in general, DCS data allow a better fit, of constant quality in the investigated temperature range. FCS data fitting is quite satisfactory as well; however, its quality decreases at low temperature. In the HFBS case, relatively high $\chi^{2}$ values are obtained; for this reason the obtained parameters have to be taken with some scrutiny. However, it is very satisfactory that the results obtained 
Table 3. Numerical values of the extracted fitting parameters from the analysis of the FCS data.

\begin{tabular}{|c|c|c|c|c|c|}
\hline \multirow[b]{2}{*}{$T(\mathrm{~K})$} & \multicolumn{5}{|c|}{ MCM-41-S (25 Å) } \\
\hline & $\tau_{0}(\mathrm{ps})$ & $\tau_{\mathrm{R}}(\mathrm{ps})$ & $\beta$ & $\gamma$ & $\chi^{2}$ \\
\hline 310 & $1.25 \pm 0.57$ & $94.1 \pm 6.4$ & $0.658 \pm 0.007$ & $2.61 \pm 0.34$ & 0.39 \\
\hline 300 & $2.12 \pm 0.10$ & $99.6 \pm 0.3$ & $0.854 \pm 0.01$ & $2.04 \pm 0.01$ & 0.56 \\
\hline 290 & $2.47 \pm 0.85$ & $138.6 \pm 20.0$ & $0.761 \pm 0.065$ & $2.13 \pm 0.27$ & 0.62 \\
\hline 280 & $2.86 \pm 0.65$ & $339.2 \pm 83.9$ & $0.701 \pm 0.001$ & $2.2 \pm 0.13$ & 1.14 \\
\hline 270 & $3.82 \pm 4.54$ & $341.6 \pm 2.2$ & $0.624 \pm 0.001$ & $2.17 \pm 1.67$ & 2.24 \\
\hline 260 & $6.44 \pm 3.28$ & $342.1 \pm 1.0$ & $0.695 \pm 0.150$ & $2.11 \pm 0.68$ & 4.54 \\
\hline \multirow[t]{2}{*}{250} & $8.83 \pm 4.80$ & $697.1 \pm 0.7$ & $0.503 \pm 0.152$ & $2.31 \pm 0.76$ & 12.73 \\
\hline & \multicolumn{5}{|c|}{ MCM-48-S (22 Å) } \\
\hline 310 & $0.86 \pm 0.56$ & $149.4 \pm 0.1$ & $0.543 \pm 0.074$ & $3.00 \pm 0.48$ & 1.20 \\
\hline 300 & $2.10 \pm 0.62$ & $63.0 \pm 0.7$ & $0.876 \pm 0.038$ & $2.07 \pm 0.23$ & 0.99 \\
\hline 290 & $2.80 \pm 0.10$ & $49.7 \pm 0.1$ & $0.737 \pm 0.069$ & $2.13 \pm 0.01$ & 1.05 \\
\hline 280 & $3.10 \pm 0.01$ & $600.9 \pm 54.1$ & $0.672 \pm 0.003$ & $2.20 \pm 0.02$ & 1.15 \\
\hline 270 & $4.71 \pm 1.20$ & $629.3 \pm 1.1$ & $0.585 \pm 0.069$ & $2.18 \pm 0.30$ & 2.92 \\
\hline
\end{tabular}

Table 4. Numerical values of the extracted fitting parameters from the analysis of the DCS data.

\begin{tabular}{|c|c|c|c|c|c|}
\hline \multirow[b]{2}{*}{$T(\mathrm{~K})$} & \multicolumn{5}{|c|}{ MCM-41-S (25 ̊) } \\
\hline & $\tau_{0}(\mathrm{ps})$ & $\tau_{\mathrm{R}}(\mathrm{ps})$ & $\beta$ & $\gamma$ & $\chi^{2}$ \\
\hline 280 & $4.52 \pm 1.68$ & $110.4 \pm 27.4$ & $0.590 \pm 0.050$ & $2.26 \pm 0.27$ & 0.85 \\
\hline 270 & $6.56 \pm 0.41$ & $336.7 \pm 1.7$ & $0.550 \pm 0.001$ & $2.29 \pm 0.08$ & 0.96 \\
\hline 260 & $11.50 \pm 3.85$ & $511.0 \pm 2.7$ & $0.530 \pm 0.001$ & $2.26 \pm 0.27$ & 1.19 \\
\hline \multirow[t]{2}{*}{250} & $19.85 \pm 7.56$ & $3704.8 \pm 487.7$ & $0.518 \pm 0.005$ & $2.22 \pm 0.30$ & 1.07 \\
\hline & \multicolumn{5}{|c|}{ MCM-48-S (22 ̊) } \\
\hline 280 & $4.20 \pm 1.39$ & $531.1 \pm 0.1$ & $0.522 \pm 0.003$ & $2.48 \pm 2.47$ & 1.42 \\
\hline 270 & $11.17 \pm 4.31$ & $138.3 \pm 0.2$ & $0.503 \pm 0.002$ & $2.24 \pm 0.29$ & 1.72 \\
\hline 260 & $34.73 \pm 14.71$ & $146.1 \pm 2.1$ & $0.526 \pm 0.001$ & $1.89 \pm 0.33$ & 3.17 \\
\hline 250 & $105.68 \pm 56.40$ & $407.3 \pm 0.7$ & $0.434 \pm 0.001$ & $2.10 \pm 0.70$ & 4.63 \\
\hline
\end{tabular}

Table 5. Numerical values of the extracted fitting parameters from the analysis of the HFBS data.

\begin{tabular}{llcllr}
\hline \multicolumn{5}{c}{ MCM-48-S (22 A) } \\
$T(\mathrm{~K})$ & $\tau_{0}(\mathrm{ps})$ & $\tau_{\mathrm{R}}(\mathrm{ps})$ & $\beta$ & $\gamma$ & $\chi^{2}$ \\
\hline 245 & $425.8 \pm 29.6$ & $5355 \pm 5$ & $0.4 \pm 0.005$ & $1.00 \pm 0.10$ & 11.58 \\
240 & $486.1 \pm 4.5$ & $48360 \pm 5$ & $0.4 \pm 0.010$ & $0.98 \pm 0.12$ & 11.19 \\
235 & $843.3 \pm 59.0$ & $166100 \pm 17$ & $0.4 \pm 0.027$ & $0.77 \pm 0.17$ & 9.52 \\
230 & $1745.4 \pm 5.4$ & $23319 \pm 2$ & $0.4 \pm 0.037$ & $0.44 \pm 0.30$ & 8.69 \\
225 & $2551.1 \pm 177.7$ & $2.10 \mathrm{E} 6 \pm 10$ & $0.4 \pm 0.05$ & $0.25 \pm 0.30$ & 8.21 \\
220 & $3536.3 \pm 7.0$ & $4.88 \mathrm{E} 6 \pm 48$ & $0.4 \pm 0.05$ & $0.07 \pm 0.23$ & 8.94 \\
\hline
\end{tabular}

using very different spectrometers agree very nicely. It is remarkable that the same model is able to describe the dynamics of the system over a range of $100 \mathrm{~K}$. 


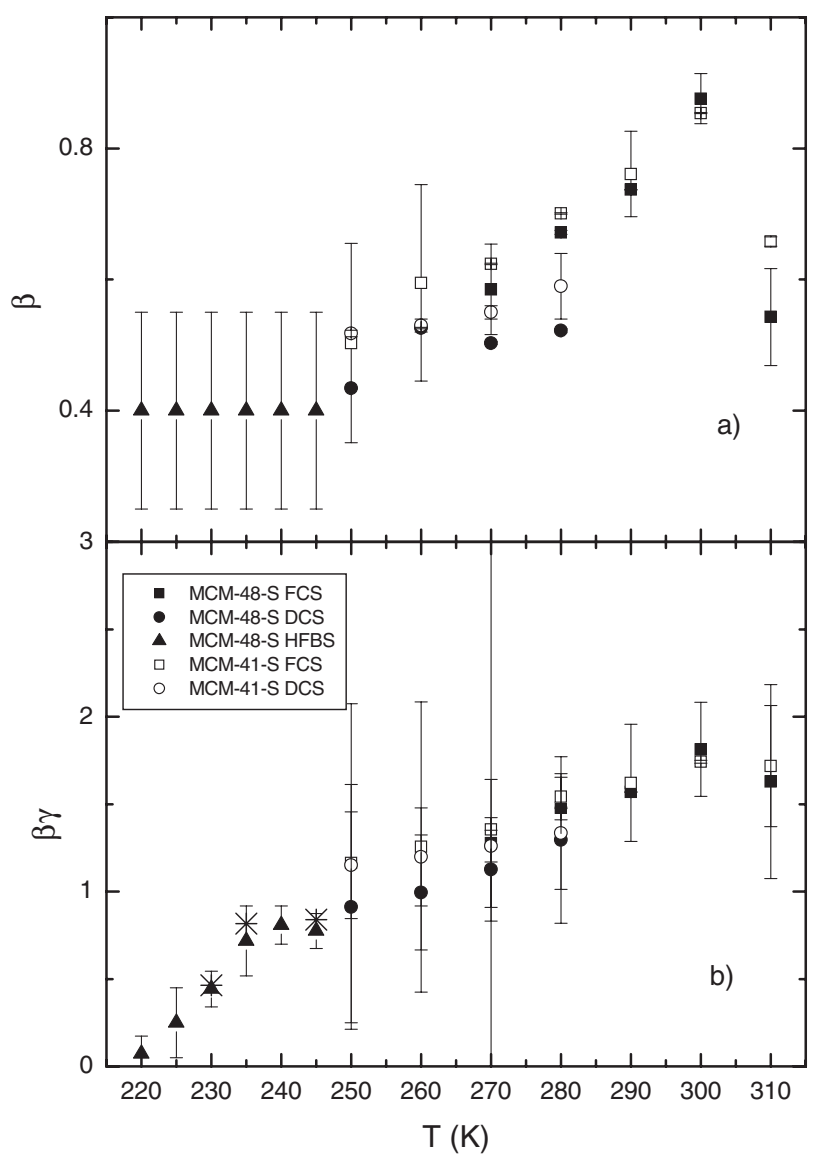

Figure 19. Temperature dependence of stretch exponent, $\beta$, and of the translational relaxation time $Q$-dependence power law exponent, $\gamma$. In panel (b) the product $\beta \gamma$ is reported. In panel (b) the asterisks are the results when beta is fixed to the value 0.4 in the analysis of HFBS data.

\section{2. $\beta, \gamma$, and $\beta \gamma$}

The fitting of the data allowed us to extract the parameters describing the translational and rotational dynamics of water. Thus, we have been able to study the effects of confinement and temperature on hydration water in MCM nanoporous sieves.

Figure 19 reports the temperature dependence of the parameters $\beta$ and $\beta \gamma$ obtained from the fitting procedure. In the previous analysis without fixing the value of $p$, we obtained values of $\beta$ increasing with decreasing temperature. We had suggested that this occurrence was most probably due to a finite resolution effect. In fact, fixing $p$ to the value corresponding to the scattering of the surface silanol groups, $\beta$ decreases with the lowering of temperature. This behaviour is in agreement with the results obtained by MD simulations and is more sound with the idea that upon supercooling the diffusive dynamics of a water molecule involves the cooperative rearrangement of the surrounding particles. This occurrence causes the nonexponentiality of the decaying function. As a matter of fact, when water is confined in nanoporous materials the translational long time relaxation is already a stretched exponential at room temperature. HFBS data, in the temperature range $245-220 \mathrm{~K}$, are consistent with a low value $(0.4)$ of $\beta$. 
It is well known that a Fickian diffusive process has a $Q^{2}$ dependence. In hydration and supercooled water, since the diffusive process is somewhat obstructed, the average translational relaxation time follows a power law in $Q$, with a power exponent $\leqslant 2$. In our previous analysis we actually found that at room temperature $\gamma$ is $\approx 2$, indicating a normal diffusive behaviour, and that it progressively decreases with the lowering of temperature.

Recently, a series of papers has investigated in detail the Gaussianity of the relaxation dynamics in polymers [62-64]. Very interesting results have been reported. In particular, many similarities between the behaviour of polymers and of supercooled liquids, water in particular, have been found. It has been reported that the product $\beta \gamma$ is two at high $Q$ and decreases above a critical $Q$ value, $Q_{\text {c }}$. This indicates the transition from a Gaussian to a non-Gaussian behaviour. In fact, $\beta \gamma$ is the power dependence of $Q$ in the ISF of the centre of mass. If $\beta \gamma=2$, the van Hove self-correlation function of the centre of mass, $G_{\mathrm{s}}^{\mathrm{CM}}(r, t)$, which is the Fourier transform of $F_{\mathrm{T}}(Q, t)$, is a Gaussian function. In water, the van Hove selfcorrelation function of the centre of mass has been investigated by MD simulations [22, 65]. Through the investigation of the non-Gaussian parameter, $\alpha_{2}$, it has been shown that $G_{\mathrm{s}}^{\mathrm{CM}}(r, t)$ is non-Gaussian at long times at supercooled temperatures.

We report the experimentally obtained values of $\beta \gamma$ in figure 19 panel (b). $\beta \gamma$ is slightly less than two and decreases with temperature in such a way that it is almost a linear function of $T$. It is very interesting that it seems to approach the Gaussian, diffusive value of two at high temperature. From the data it can be argued that water dynamics is Gaussian at ambient temperature and that it increasingly deviates from the Gaussian behaviour as temperature is lowered. It is noteworthy that the values of $\beta \gamma$ from all three spectrometers agree very well with each other. Even if, as far as $\beta$ and $\gamma$ are concerned, no difference can be noticed between MCM-41-S (25 $\AA$ ) and MCM-48-S (22 $\AA$ ), it can be argued that the obtained $\beta \gamma$ values are slightly higher in MCM-41-S (25 $\AA$ ). This finding could be related to the fact that the pores of this matrix are slightly bigger. It is worth noting that in MCM-48-S (22 $\AA$ ) $\beta \gamma$ seems to approach zero at $T=220 \mathrm{~K}$, indicating the complete arrest of the diffusional motion at this temperature. We should qualify this statement, allowing for finite resolution of the instrument. When the relaxation time approaches the limit of $\approx 1 \mathrm{~ns}$, the dynamics is frozen on the timescale of the backscattering instrument.

\section{3. $\left\langle\tau_{0}\right\rangle,\left\langle\tau_{\mathrm{R}_{1}}\right\rangle$ and $\left\langle\tau_{\mathrm{R}_{2}}\right\rangle$}

In figures 20-22, we show the temperature dependence of the average translational relaxation time, $\left\langle\tau_{0}\right\rangle=\left(\tau_{0} / \beta\right) \Gamma(1 / \beta)$, as obtained from the experimental data. In the case of MCM-41-S (25 $\AA$ ) and MCM-48-S (22 A), no clear critical slowing down can be seen in our experimental data $(220 \mathrm{~K} \leqslant T \leqslant 310 \mathrm{~K})$, nor could an obvious fit be furnished with the Vogel-FulcherTamman law. However, an Arrhenius law $\left\langle\tau_{0}\right\rangle \sim \exp \left(E_{\mathrm{A}} / R T\right)$, where $E_{\mathrm{A}}=11.3$ and $7.73 \mathrm{kcal} \mathrm{K}^{-1} \mathrm{~mol}^{-1}$ in the case of MCM-48-S and MCM-41-S respectively, furnishes the best fit to the data. Even if these findings cannot be considered conclusive, two possible scenarios seem to apply: inside small nanoporous materials the dynamics of water is affected in such a way that either the critical temperature is shifted to a lower temperature or no critical behaviour is effective. MD simulations of SPC/E water confined in a $40 \AA$ diameter Vycor tube have found a lowering of the critical temperature of about $30 \mathrm{~K}$ with respect to the findings on bulk SPC/E water [27]. Therefore, MD simulations support the first hypothesis. In order to clarify this very important issue, we collected data on water confined in MCM-41-S matrices with $18 \AA$ (mac-1219-7) and $14 \AA$ (mac-0214-4). Using these samples, we can distinguish a quasielastic broadening down to $200 \mathrm{~K}$, indicating that we can investigate supercooled states of water at temperatures lower than those accessible with the previous samples. We report here 


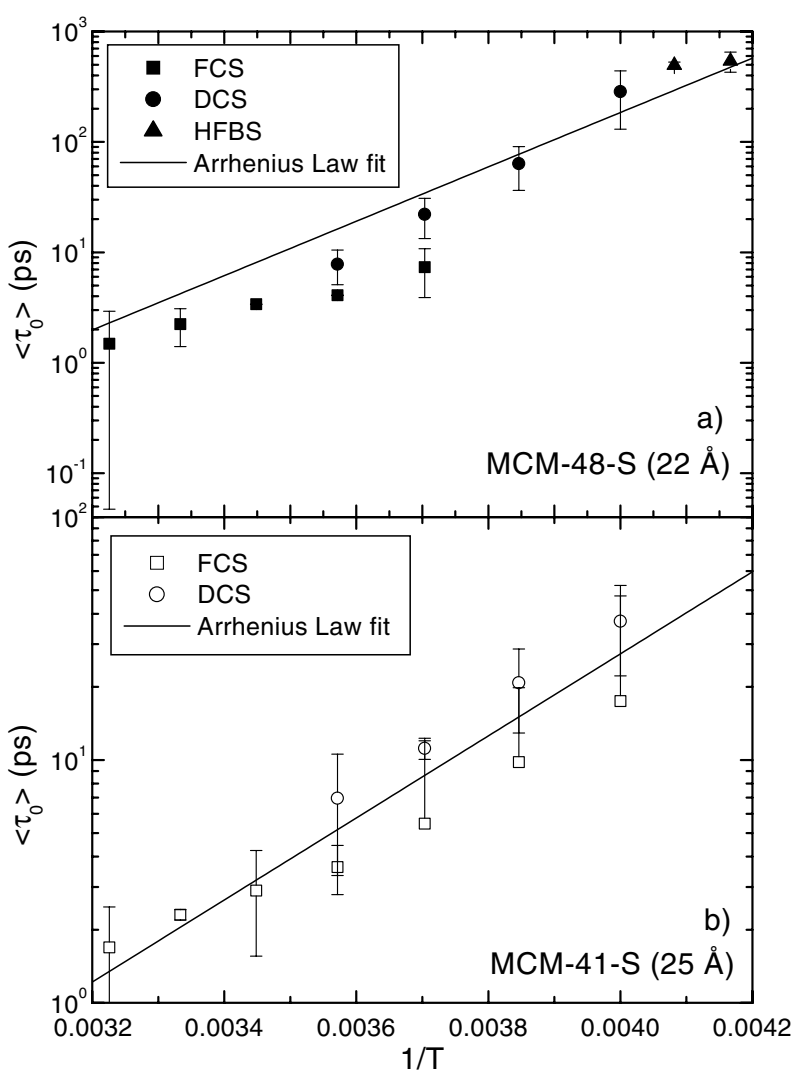

Figure 20. Temperature dependence of the average translational relaxation time as extracted from the analysis of the data taken using the three QENS spectrometers FCS, DCS, and HFBS. Panels (a) and (b) show the results for the MCM-48-S (22 A) and MCM-41-S (25 ̊) matrices, respectively. The open and solid points stand for the experimental data. Continuous lines are fit to the data according to Arrhenius law, $\left\langle\tau_{0}\right\rangle \sim \exp \left(E_{\mathrm{A}} / R T\right)$, where $E_{\mathrm{A}}=11.3$ and $7.73 \mathrm{kcal} \mathrm{K}^{-1} \mathrm{~mol}^{-1}$ in the case of MCM-48-S and MCM-41-S, respectively.

some preliminary results $\left(\left\langle\tau_{0}\right\rangle\right.$ versus $T$, figure 21$)$ because we regard them as very interesting for the scientific community. We eliminated the solid points in figure 21 and redrew the figure in figure 22 with a $\left\langle\tau_{0}\right\rangle$ versus $1 /\left(T-T_{0}\right)$ scale. As shown in figures 21 and 22 , we find that in the range $325-225 \mathrm{~K}$ a VFT law with $T_{0}=200.5 \mathrm{~K}$ (mac-0124-4, $14 \AA$ ) and $206.2 \mathrm{~K}$ (mac1219-7, $18 \AA$ ) can describe the experimental data very well. These results suggest an ideal glass transition temperature, $T_{0} \approx 200 \mathrm{~K}$, which is in accordance with the temperature from $\mathrm{MD}\left(T_{0} \approx 170 \mathrm{~K}\right)$ since MD simulation data show an about $27 \mathrm{~K}$ lower value than real data. In figure 21 , it is noteworthy that at $T>220 \mathrm{~K}\left\langle\tau_{0}\right\rangle$ follows the VFT law, whereas for $T \leqslant 220 \mathrm{~K}$ $\left\langle\tau_{0}\right\rangle$ diverges from the VFT law, indicating the existence of a glass transition (structural arrest transition) at $T=220 \mathrm{~K}$. It is also very noteworthy that, in the whole temperature range, as temperature decreases $\left\langle\tau_{0}\right\rangle$ increases by about five orders of magnitude, indicating a dramatic slowing down of translational dynamics of water in MCM-41-S.

In order to study the effect of the confinement on water dynamics, we compare the obtained translational relaxation times with the data reported for bulk water. At $T=300 \mathrm{~K}, \beta$ is only slightly less than unity, $\gamma \approx 2$, and $\beta \gamma \approx 2$; therefore, we can calculate, with a good approximation, the diffusion coefficient as $D=a^{2} / \tau_{0}$. In bulk water, from NMR [66], it 


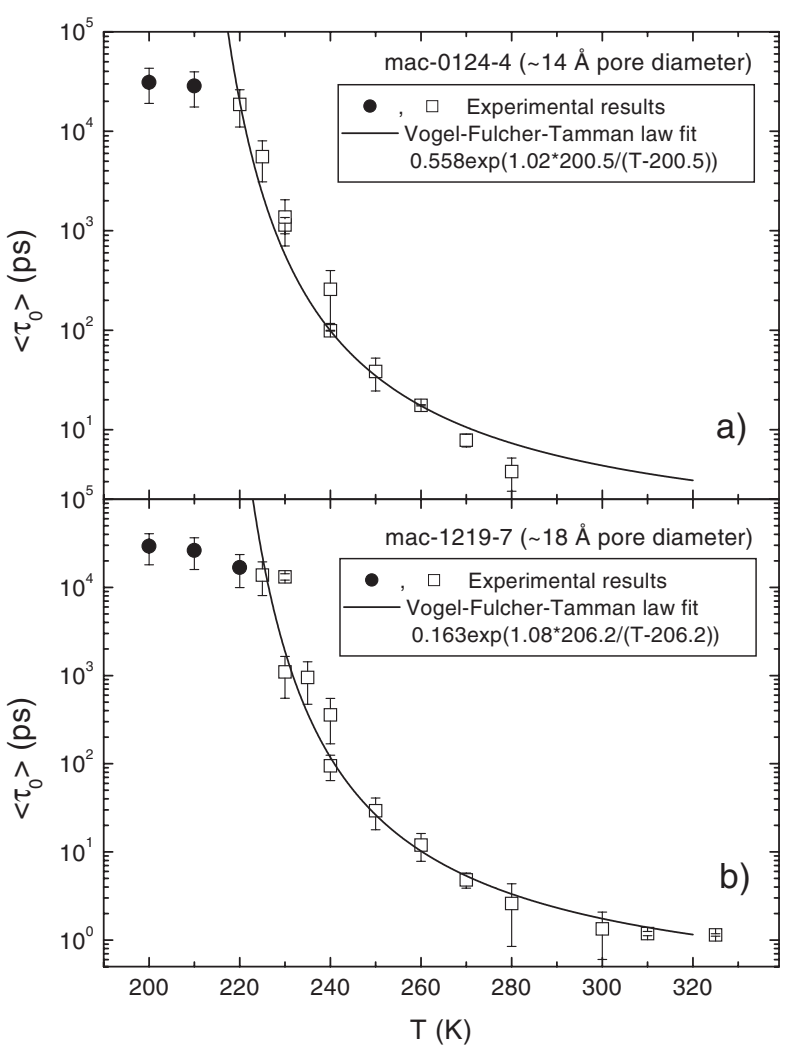

Figure 21. Temperature dependence of the average translational relaxation time as extracted from the analysis of the data taken using the two spectrometers DCS and HFBS. Panels (a) and (b) show the results for the MCM-41-S (mac-0124-4) and MCM-41-S (mac-1219-7) matrices, respectively. Continuous curves are fits to the data according to the Vogel-Fulcher-Tamman law. Solid points have not been considered for the fit.

has been found that $D=2.41 \times 10^{-5} \mathrm{~cm}^{2} \mathrm{~s}^{-1}$; we find $D=1.11 \times 10^{-5} \mathrm{~cm}^{2} \mathrm{~s}^{-1}$ and $D=1.08 \times 10^{-5} \mathrm{~cm}^{2} \mathrm{~s}^{-1}$ in the MCM-48-S (22 $\AA$ ) and MCM-41-S (25 $\AA$ ) matrices, respectively. Then, the effective slowing down of the translational dynamics is about 2.2fold. At $T=280 \mathrm{~K}$, in bulk water, from NMR, $D=1.39 \times 10^{-5} \mathrm{~cm}^{2} \mathrm{~s}^{-1}$. Although the derivation of the diffusion coefficient is not exact since $\beta \gamma$ is not equal to two, at $T=280 \mathrm{~K}$ we obtain $D=0.32 \times 10^{-5} \mathrm{~cm}^{2} \mathrm{~s}^{-1}$ and $D=0.36 \times 10^{-5} \mathrm{~cm}^{2} \mathrm{~s}^{-1}$ in the MCM-48-S and MCM-41-S matrices, respectively, as obtained from the DCS data. At lower temperatures the effective slowing down increases to a factor $\approx 4$. We note that the fixing of the parameter $p$ slightly affects the obtained values for $\left\langle\tau_{0}\right\rangle$, as can be seen comparing these results with those previously published [49].

The effect of the pore size can be studied by comparing our data with the results of a previous work on water confined in a Vycor glass with average pore diameter of $50 \AA$, in which the data have been analysed using the translational RCM [31]. We simply compare the average translational relaxation times at $Q=0.46 \AA^{-1}$; at this low $Q$ value rotational contributions are negligible. In Vycor $\left\langle\tau_{\mathrm{T}}\right\rangle\left(Q=0.46 \AA^{-1}\right)=46.90,82.43,109.49$, and $158.41 \mathrm{ps}$, at $T=293$, 278,268 , and $258 \mathrm{~K}$ respectively. In MCM-41-S $(25 \AA)$ we find $\left\langle\tau_{\mathrm{T}}\right\rangle\left(Q=0.46 \AA^{-1}\right)=66.5$, 192.6, 323.3, and 575.4 ps; in MCM-48-S we find $\left\langle\tau_{\mathrm{T}}\right\rangle\left(Q=0.46 \AA^{-1}\right)=77.4$, 297.9, 




Figure 22. The plot of the average translational relaxation time. Panels (a) and (b) show the results for the MCM-41-S (mac-0124-4) and MCM-41-S (mac-1219-7) matrices, respectively. Continuous lines are fits to the data according to the Vogel-Fulcher-Tamman law.

594.4, and $1021.6 \mathrm{ps}$ at $T=290,280,270$, and $260 \mathrm{~K}$, respectively, as obtained from FCS $(T=290 \mathrm{~K})$ and DCS data. Therefore, in these MCM matrices, whose pore diameters are half of that of Vycor glass, the translational dynamic of water is, depending on temperature and on the confining matrix, from $\sim 1.5$ to $\sim 6.5$ times slower than that of water in Vycor. These findings show that the translational dynamics of water is strongly slowed down in pores with such small dimensions.

As far as the rotational dynamics is concerned, the average relaxation time of the first-, $\left\langle\tau_{\mathrm{R}_{1}}\right\rangle$, and second-, $\left\langle\tau_{\mathrm{R}_{2}}\right\rangle$,-order rotational correlation functions can be measured using dielectric relaxation spectroscopy and NMR respectively [67]. In particular, it has been reported that $\left\langle\tau_{\mathrm{R}_{2}}\right\rangle$ follows a critical behaviour with a critical temperature very close to $T_{\mathrm{c}}$. Figure 8 shows that the MD generated data obey a critical law with $T_{\mathrm{c}}^{\mathrm{MD}}=204 \mathrm{~K}$.

In figure 8 inset (b), we show the $\beta$ values for the fitting of $C_{1}(t)$ and $C_{2}(t)$ as open squares and triangles, respectively. In the same panel, we report as dots the values for the translational dynamics. Within the error limit they agree, confirming the validity of the assumption, in the fitting algorithm, that $\beta \approx \beta_{\mathrm{R}_{1}}$.

Figure 23 shows the average rotational relaxation time, $\left\langle\tau_{\mathrm{R}}\right\rangle=\left(\tau_{\mathrm{R}} / \beta\right) \Gamma(1 / \beta)$, as obtained from the experimental results. In the temperature range $220 \mathrm{~K}<T<310 \mathrm{~K}$, the average rotational relaxation time increases five orders of magnitude, showing a dramatic slowing down of the rotational dynamics. However, we could not succeed in fitting the data to a critical law or VFT law. These results are strongly affected by finite resolution effects. 


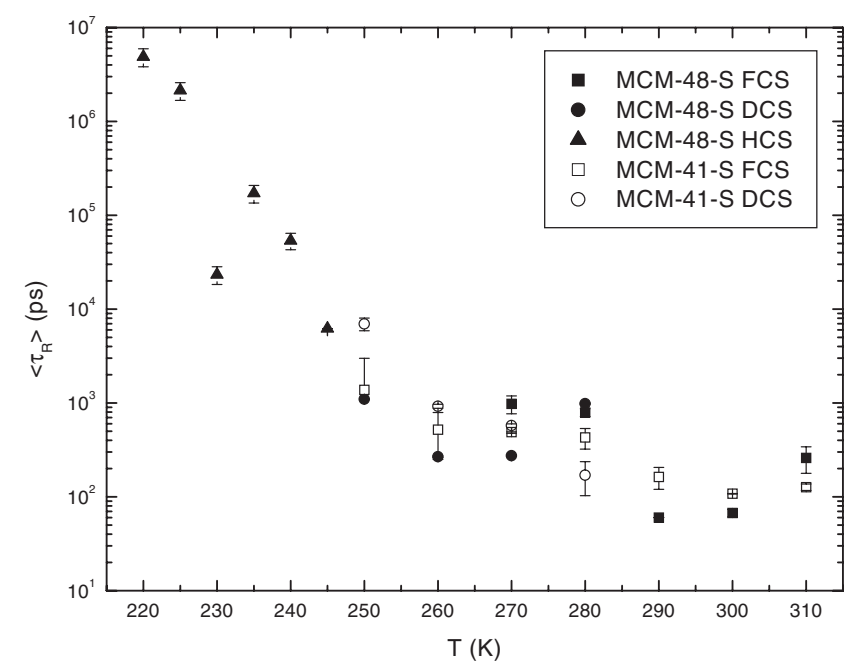

Figure 23. Temperature dependence of the average rotational relaxation time as extracted from the analysis of the data taken using the three QENS spectrometers FCS, DCS, and HFBS. Solid and open scatter show the results for the MCM-48-S (22 ̊) and MCM-41-S (25 ̊) matrix respectively.

Figure 23 compares the average rotational relaxation times for the two matrices. The size and morphology of the pores do not seem to affect the rotational dynamics, as the obtained data can hardly be considered different for the two samples. In fact, the rotation process of a water molecule takes place within a sphere of radius $b$ from the centre of mass, and is not directly affected by confinement within pores larger than $20 \AA$. However, it is reasonable to presume that confinement affects the rotational dynamics through translational-rotational coupling. In this case the pore dimensions are quite similar and $\left\langle\tau_{R}\right\rangle$ values in the two matrices tend to coincide.

\section{Conclusion}

Within the decoupling approximation (which is accurate to $\leqslant 0.09$ ), the relaxing cage model for the ISF of hydrogen atoms in water can be used to fit both MD and QENS data for deeply supercooled water successfully. We have also compared the behaviour of water inside two matrices with different morphologies. The small dimensions of the pores $(\approx 20 \AA)$ allowed us to investigate the temperature region below the homogeneous nucleation temperature $\left(T_{\mathrm{H}}=235 \mathrm{~K}\right)$, obtaining information on the dynamics of water at temperatures rarely investigated experimentally. Furthermore, the dynamics of water confined in nanoporous silica materials has been shown to be similar to that of a supercooled bulk water.

Using three different QENS spectrometers, water in MCM-41-S with pore sizes $25 \AA$, $18 \AA$, and $14 \AA$ and MCM-48-S with a pore size $22 \AA$ has been studied in a wide range, from room temperature to the deeply supercooled state. By fitting QENS data with the RCM, one can extract both the $Q$-dependent average translational relaxation time $\tau_{\mathrm{T}}(Q)$ and $Q$-independent average rotational relaxation times $\tau_{\mathrm{R}_{1}}$ and $\tau_{\mathrm{R}_{2}}$ as a function of temperature. The $Q$ dependence of the average translational relaxation time follows a power-law $\tau_{\mathrm{T}}(Q)=\tau_{0}(a Q)^{-\gamma}$, $\left\langle\tau_{0}\right\rangle=\left(\tau_{0} / \beta\right) \Gamma(1 / \beta)$. The area of the elastic component $p$, mainly contributed from the hydrogen atoms in the surface silanol groups, is fixed to its plateau value for each sample. At room temperature the exponents $\beta \approx 1$ and $\gamma \approx 2$ and both exponents decrease with 
temperature in such a way that $\beta$ decreases to a plateau value of 0.4 and $\beta \gamma$ is a linear function of $T$. The results of the most recent studies show that $\left\langle\tau_{0}\right\rangle$ follows the Vogel-Fulcher-Tamman law with an ideal glass transition temperature $T_{0} \approx 200 \mathrm{~K}$ for MCM-41-S with $14 \AA$ and $18 \AA$ pores. This temperature is about the same as the ideal glass transition temperature for bulk water from MD simulation. The average rotational relaxation time does not follow the VFT law but it increases by five orders of magnitude within this temperature range.

Inside the investigated MCM-41-S and MCM-48-S matrices the translational and rotational dynamics of water are substantially slowed down with respect to bulk water. Meanwhile, the effect is enhanced as temperature decreases. An exponential increase of the average relaxation times of translational and rotational dynamics is detected on lowering temperature. Both the average translational and rotational relaxation times slow down tremendously as the confined water is supercooled and the dimensionality of the diffusion process decreases in a way that strongly indicates the existence of a glass transition (structural arrest transition) temperature at $220 \mathrm{~K}$.

The comparison of the two matrices (MCM-41-S with pore size $25 \AA$ and MCM-48-S with pore size $22 \AA$ ) suggests that the rotational dynamics is not strongly influenced by the morphology of the pores. On the other hand, it is not clear whether the different translational dynamics of water in MCM-41-S and MCM-48-S should be attributed to the morphology of the pore or to their slightly different dimensions.

\section{Acknowledgments}

We are grateful to Dr John R Copley, Craig Brown, Zema Chowdhuri, and Robert Dimeo for generous help during the experiments. Research at MIT is supported by DE-FG02-90ER45429 and 2113-MIT-DOE-591. This work utilized facilities supported in part by the National Science Foundation under Agreement No DMR-0086210. Identification of a commercial product does not imply recommendation or endorsement by the National Institute of Standards and Technology, nor does it imply that the product is necessarily the best for the stated purpose.

\section{References}

[1] Debenedetti P G 1997 Metastable Liquids: Concepts and Principles (Princeton, NJ: Princeton University Press)

[2] Sciortino F, Nave E L and Tartaglia P 2003 Phys. Rev. Lett. 91155701

[3] Bertolini D and Tani A 1995 Phys. Rev. E 511091

[4] Speedy R J and Angell C A 1976 J. Chem. Phys. 65851

[5] Chen S-H and Bellissent-Funel M-C 1994 Hydrogen Bond Networks (NATO ASI Series C: Mathematical and Physical Sciences vol 435) ed M-C Bellissent-Funel and J C Dore (Dordrecht: Kluwer-Academic) p 289

[6] Benham M J, Cook J C, Li J-C, Ross D K, Hall P L and Sarkissian B 1989 Phys. Rev. B 39633

[7] Botti A, Bruni F, Isopo A, Ricci M A and Soper A K 2002 J. Chem. Phys. 1176196

[8] Rønne C, Åstrand P-O and Keiding S R 1999 Phys. Rev. Lett. 822888

[9] Rønne C and Keiding S R 2002 J. Mol. Liq. 101199

[10] Bergman R and Swenson J 2000 Nature 403283

[11] Bergman R, Swenson J, Børjesson L and Jacobsson P 2000 J. Chem. Phys. 113357

[12] Ryabov Y, Gutina A, Archipov V and Feldman Y 2001 J. Phys. Chem. B 1051845

[13] Gutina A, Rysiakiewicz-Pasek E and Feldman Y 2001 Glass Phys. Chem. 27394

[14] Gutina A, Antropova T, Rysiakiewicz-Pasek E, Virnik K and Feldman Y 2003 Micropor. Mesopor. Mater. 58 237

[15] D’Orazio F, Bhattacharja S, Halperin W P and Gerhardt R 1989 Phys. Rev. Lett. 6343

[16] D’Orazio F, Bhattacharja S, Halperin W P, Eguchi K and Mizusaki T 1990 Phys. Rev. B 429810

[17] Stapf S, Kimmich R and Niess J 1994 J. Appl. Phys. 75529

[18] Stapf S, Kimmich R and Seitter R-O 1995 Phys. Rev. Lett. 752855

[19] Korb J P, Malier J-P L, Cros F, Xu S and Jonas J 1996 Phys. Rev. Lett. 772312 
[20] Holly R, Peemoeller H, Choi C and Pintar M M 1998 J. Chem. Phys. 1084183

[21] Gallo P, Sciortino F, Tartaglia P and Chen S-H 1996 Phys. Rev. Lett. 762730

[22] Sciortino F, Gallo P, Tartaglia P and Chen S-H 1996 Phys. Rev. E 546331

[23] Chen S-H, Liao C, Sciortino F, Gallo P and Tartaglia P 1999 Phys. Rev. E 596708

[24] Liu L, Faraone A and Chen S-H 2002 Phys. Rev. E 65041506

[25] Chen S-H, Gallo P, Sciortino F and Tartaglia P 1997 Phys. Rev. E 564231

[26] Gallo P, Rovere M and Spohr E 2000 Phys. Rev. Lett. 854317

[27] Gallo P, Rovere M and Spohr E 2000 J. Chem. Phys. 11311324

[28] Lee S H and Rossky P J 1994 J. Chem. Phys. 1003334

[29] Zanotti J-M, Bellissent-Funel M-C and Chen S-H 2004 Water dynamics in the 'no man's land' temperature regime Phys. Rev. E submitted

[30] Bellissent-Funel M-C, Chen S-H and Zanotti J M 1995 Phys. Rev. E 514558

[31] Zanotti J M, Bellissent-Funel M-C and Chen S-H 1999 Phys. Rev. E 593084

[32] Bellissent-Funel M-C, Longeville S, Zanotti J M and Chen S-H 2000 Phys. Rev. Lett. 853644

[33] Crupi V, Majolino D, Migliardo P and Venuti V 2000 J. Phys. Chem. A 10411000

[34] Crupi V, Majolino D, Migliardo P and Venuti V 2002 J. Phys. Chem. B 10610884

[35] Swenson J, Bergman R and Howells W S 2000 J. Chem. Phys. 1132873

[36] Swenson J, Bergman R and Longeville S 2001 J. Chem. Phys. 11511299

[37] Takamuku T, Yamagami M, Wakita H, Masuda Y and Yamaguchi T 1997 J. Phys. Chem. B 1015730

[38] Mansour F, Dimeo R M and Peemoeller H 2002 Phys. Rev. E 66041307

[39] Mitra S, Mukhopadhyay R, Tsukushi I and Ikeda S 2001 J. Phys.: Condens. Matter 138455

[40] Takahara S, Nakano M, Kittaka S, Kuroda Y, Mori T, Hamano H and Yamaguchi T 1999 J. Phys. Chem. B 103 5814

[41] Gallo P, Sciortino F, Tartaglia P and Chen S-H 1996 Phys. Rev. Lett. 762730

[42] Steinhauser O 1982 Mol. Phys. 45335

[43] Berendsen H J C et al 1984 J. Chem. Phys. 813684

[44] Leuthesser E 1984 Phys. Rev. A 292765

[45] Bengtzelius U, Gotze W and Sjolander A 1984 J. Phys. C: Solid State Phys. 175915

[46] Fuchs M, Hofacker I and Latz L 1992 Phys. Rev. A 45898

[47] Kob W and Andersen H C 1995 Phys. Rev. E 514626

[48] Faraone A, Chen S-H, Fratini E, Baglioni P, Liu L and Brown C 2002 Phys. Rev. E 65040501

[49] Faraone A, Liu L, Mou C-Y, Shih P-C, Copley J R D and Chen S-H 2003 J. Chem. Phys. 1193963

[50] Faraone A, Fratini E, Baglioni P and Chen S-H 2004 Quasielastic and inelastic neutron scattering on hydrated calcium silicate pastes J. Chem. Phys. submitted

[51] Lovesey S W 1984 Theory of Neutron Scattering from Condensed Matter (Oxford: Clarendon)

[52] Teixeira J, Bellissent-Funel M-C, Chen S-H and Dianoux A J 1985 Phys. Rev. A 311913

[53] Larsson K E 1965 Thermal Neutron Scattering ed P A Egelstaff (London: Academic) p 347

[54] Sears V F 1967 Can. J. Phys. 45237

[55] Berne B J, Pechukas P and Harp G D 1968 J. Chem. Phys. 493125

[56] Squires G L 1978 Introduction to the Theory of Thermal Neutron Scattering (Cambridge: Cambridge University Press)

[57] Weinrub Allen S 1971 Cold neutron scattering by molecular liquids PhD Thesis Harvard University

[58] Fabbian L, Sciortino F and Tartaglia P 1998 J. Non-Cryst. Solids 235-237 325

[59] Shih P-C, Lin H-P and Mou C-Y 2003 Stud. Surf. Sci. Catal. 146557

[60] Liu Y, Zhang W and Pinnavaia T J 2000 J. Am. Chem. Soc. 1228791

[61] Schumacher K, Ravikovitch P I, Du Chesne A, Neimark A V and Unger K K 2000 Langmuir 164648

[62] Colmenero J, Alvarez F and Arbe A 2002 Phys. Rev. E 65041804

[63] Arbe A, Colmenero J, Alvarez F, Monkenbusch M, Richter D, Farago B and Frick B 2002 Phys. Rev. Lett. 89 245701

[64] Colmenero J, Arbe A, Alvarez F, Monkenbusch M, Richter D, Farago B and Frick B 2003 J. Phys.: Condens. Matter 15 S1127

[65] Faraone A, Liu L and Chen S-H 2003 J. Chem. Phys. 1196302

[66] Prielmeier F X, Lang E W, Speedy R J and Lüdemann H D 1988 Ber. Bunsenges. Phys. Chem. 921111

[67] Chen S-H 1991 Hydrogen Bonded Liquids (NATO ASI Series C: Mathematical and Physical Sciences vol 329) ed J C Dore and J Teixeira (Dordrecht: Kluwer-Academic) p 289 